\title{
Fingerprints of Kitaev physics in the magnetic excitations of honeycomb iridates
}

\author{
A. Revelli, ${ }^{1}$ M. Moretti Sala, ${ }^{2,3}$ G. Monaco, ${ }^{4}$ C. Hickey, ${ }^{5}$ P. Becker, ${ }^{6}$ F. Freund, ${ }^{7}$ A. Jesche, ${ }^{7}$ P. Gegenwart, ${ }^{7}$ \\ T. Eschmann, ${ }^{5}$ F. L. Buessen, ${ }^{5}$ S. Trebst,${ }^{5}$ P. H. M. van Loosdrecht, ${ }^{1}$ J. van den Brink,,${ }^{8,9}$ and M. Grüninger ${ }^{1}$ \\ ${ }^{1}$ II. Physikalisches Institut, Universität zu Köln, Zülpicher Strasse 77, D-50937 Köln, Germany \\ ${ }^{2}$ European Synchrotron Radiation Facility, BP 220, F-38043 Grenoble Cedex, France \\ ${ }^{3}$ Dipartimento di Fisica, Politecnico di Milano, Piazza Leonardo da Vinci 32, I-20133 Milano, Italy \\ ${ }^{4}$ Dipartimento di Fisica, Università di Trento, via Sommarive 14, 38123 Povo (TN), Italy \\ ${ }^{5}$ Institut für Theoretische Physik, Universität zu Köln, Zülpicher Strasse 77, D-50937 Köln, Germany \\ ${ }^{6}$ Abteilung Kristallographie, Institut für Geologie und Mineralogie, Zülpicher Strasse 49b, D-50674 Köln, Germany \\ ${ }^{7}$ Experimental Physics VI, Center for Electronic Correlations and Magnetism, University of Augsburg, 86159 Augsburg, Germany \\ ${ }^{8}$ Institute for Theoretical Solid State Physics, IFW Dresden, Helmholtzstrasse 20, 01069 Dresden, Germany \\ ${ }^{9}$ Institut für Theoretische Physik and Würzburg-Dresden Cluster of Excellence ct.qmat, \\ Technische Universität Dresden, 01062 Dresden, Germany
}

(Received 31 May 2019; revised 27 August 2020; accepted 11 September 2020; published 16 October 2020)

\begin{abstract}
In the quest for realizations of quantum spin liquids, the exploration of Kitaev materials (spin-orbit-entangled Mott insulators with strong bond-directional exchanges) has taken center stage. However, in these materials the local spin-orbital $j=1 / 2$ moments typically show long-range magnetic order at low temperature, thus defying the formation of a spin-liquid ground state. It is an intriguing question how far the properties of the Kitaev spin liquid survive in these materials despite the presence of long-range magnetic order. Using resonant inelastic $\mathrm{x}$-ray scattering (RIXS), we here report on a proximate spin-liquid regime with distinctive fingerprints of Kitaev physics in the magnetic excitations of the honeycomb iridates $\alpha-\mathrm{Li}_{2} \mathrm{IrO}_{3}$ and $\mathrm{Na}_{2} \mathrm{IrO}_{3}$. We observe a broad continuum of magnetic excitations and prove its magnetic character via the resonance behavior. The continuum is insensitive to temperature up to about $90 \mathrm{~K}$ and persists up to at least $300 \mathrm{~K}$, more than an order of magnitude larger than the magnetic ordering temperatures. This striking temperature dependence of the RIXS intensity agrees with the behavior of the nearest-neighbor spin-spin correlations of the Kitaev model. This comparison is motivated by momentum-dependent RIXS measurements of the dynamical structure factor for energies within the continuum which show that dynamical spin-spin correlations are restricted to nearest neighbors, which is a key property of the Kitaev model. Notably, these spectroscopic observations are also present in the magnetically ordered state for excitation energies above the conventional magnon excitations. Phenomenologically, our data agree with inelastic neutron scattering results on the related honeycomb compound $\alpha-\mathrm{RuCl}_{3}$, establishing a common ground for a proximate Kitaev spin-liquid regime in these materials.
\end{abstract}

DOI: 10.1103/PhysRevResearch.2.043094

\section{INTRODUCTION}

Spin liquids are elusive states of quantum matter in which quantum fluctuations prevent the formation of long-range magnetic order even at $T=0$ [1]. Theory distinguishes various types of spin liquids, e.g., gapless and gapped variants with topological order [2,3]. A particularly interesting version arises from exchange frustration as described by the Kitaev model on tricoordinated lattices [4,5], which exhibits fractional excitations in the form of itinerant Majorana fermions and static visons [6]. Realizations of such Kitaev spin liquids are sought in Mott insulators with spin-orbit-entangled

Published by the American Physical Society under the terms of the Creative Commons Attribution 4.0 International license. Further distribution of this work must maintain attribution to the author(s) and the published article's title, journal citation, and DOI. $j=1 / 2$ moments in edge-sharing bonding geometry [7] such as the honeycomb iridates $\mathrm{H}_{3} \mathrm{LiIr}_{2} \mathrm{O}_{6}$ [8] and $\mathrm{Cu}_{2} \mathrm{IrO}_{3}$ [9] which do not show magnetic order down to at least $50 \mathrm{mK}$ and $260 \mathrm{mK}$, respectively [10]. The related compounds $\alpha-\mathrm{Li}_{2} \mathrm{IrO}_{3}$, $\mathrm{Na}_{2} \mathrm{IrO}_{3}$, and $\alpha-\mathrm{RuCl}_{3}$ all show dominant bond-directional Kitaev interactions [11-13] but order magnetically below 7$15 \mathrm{~K}$, which is commonly attributed to additional coupling terms, e.g., Heisenberg and off-diagonal interactions [12-14].

This raises the question of whether these materials are, by virtue of their dominant bond-directional interactions, destined to form Kitaev spin liquids, which however is impeded at low temperatures by spurious additional couplings. This situation can be compared to quasi-1D quantum spin systems such as $\mathrm{KCuF}_{3}$ showing $3 \mathrm{D}$ long-range order at low temperature due to a residual interchain coupling. Despite magnetic ordering, the excitation spectrum can, however, still be described by a continuum of fractional spinons for energies larger than the energy scale of the interchain coupling [15], with the low-energy magnons taking only a small fraction of the total 


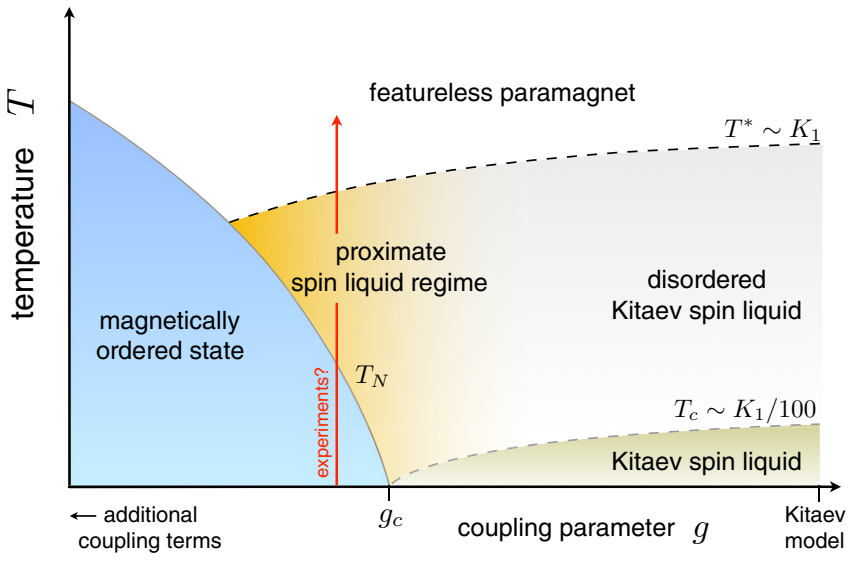

FIG. 1. Proximate spin-liquid regime in a hypothesized phase diagram of the Kitaev model in the presence of additional magnetic interactions. In the pure Kitaev model (right), the ordered $Z_{2}$ quantum-spin-liquid ground state is separated from the featureless, high-temperature paramagnet by a disordered $Z_{2}$ spin liquid (dashed lines denote crossovers). Long-range magnetic order is expected for sufficiently large strength of additional exchange terms (left). Above the magnetic-ordering transition, an intermediate-temperature regime might persist, in which the system resides in a "proximate spin-liquid regime," where it exhibits fingerprints of Kitaev physics up to temperatures of the order of the Kitaev coupling $K_{1}$.

spectral weight. At high energy, the spinon continuum is not affected by the ordering temperature $T_{N}$ and persists to temperatures far above $T_{N}$. For the Kitaev materials at hand, this suggests the existence of a proximate spin-liquid regime above the long-range ordering temperature in which the physics of fractionalized excitations manifests itself; see Fig. 1. Indeed, $\alpha-\mathrm{RuCl}_{3}$ has been described as a proximate spin liquid based on inelastic neutron scattering data $[16,17]$ which revealed a continuum of magnetic excitations, smeared out both in energy and momentum space, which persists far above the Néel temperature $T_{N}$. The large width in energy is attributed to the fractional character of excitations; the sinusoidal momentum dependence of the dynamical structure factor reflects the dominance of nearest-neighbor correlations. In general, an excitation continuum based on short-range correlations counts as a clear fingerprint of spin-liquid behavior as reported in inelastic neutron scattering on the kagome lattice antiferromagnet herbertsmithite [18] and $\mathrm{Ca}_{10} \mathrm{Cr}_{7} \mathrm{O}_{28}$ [19]. Note that the strict restriction to nearest-neighbor correlations is a key property of the Kitaev model. The proximity of $\alpha-\mathrm{RuCl}_{3}$ to a Kitaev spin liquid was most prominently highlighted by the observation of a half-integer quantized thermal Hall effect attributed to fractionalized Majorana fermions in an external magnetic field suppressing long-range order [20,21]. Also a low-energy Raman continuum was attributed to Majorana fermions [22,23].

Here, we address the question of whether the existence of a proximate spin-liquid regime is a generic feature of $\mathrm{Ki}$ taev materials by exploring the $j=1 / 2$ honeycomb iridates $\mathrm{Na}_{2} \mathrm{IrO}_{3}$ and $\alpha-\mathrm{Li}_{2} \mathrm{IrO}_{3}$. According to ab initio calculations [13], the iridates and in particular $\mathrm{Na}_{2} \mathrm{IrO}_{3}$ are expected to be closer to the pure Kitaev limit than $\alpha-\mathrm{RuCl}_{3}$. In $\mathrm{Na}_{2} \mathrm{IrO}_{3}$, experimental evidence for dominant Kitaev coupling stems from resonant elastic x-ray scattering [11]. However, experimental studies of the magnetic excitations in the honeycomb iridates are challenging and thus scarce. Powder inelastic neutron scattering data of $\mathrm{Na}_{2} \mathrm{IrO}_{3}$ and $\alpha-\mathrm{Li}_{2} \mathrm{IrO}_{3}$ show magnon modes at a few meV [24,25], but further investigations are obstructed by the strong neutron absorption of Ir. Resonant inelastic x-ray scattering (RIXS) has been used to study the magnetic excitations of corner-sharing iridates [26-30] but the much smaller energy scale of edge-sharing honeycomb iridates is comparable to the state-of-the-art energy resolution of $24 \mathrm{meV}$ [31,32]. In $\mathrm{Na}_{2} \mathrm{IrO}_{3}$, a broad inelastic RIXS feature peaking at about 30 to $50 \mathrm{meV}$ was previously attributed to the sum of magnons and phonons [33].

To explore the existence of a proximate spin-liquid regime in these honeycomb iridates we probe their magnetic excitations using RIXS. We resolve the character of the low-lying excitations by comparing $\mathrm{Na}_{2} \mathrm{IrO}_{3}$ and $\alpha-\mathrm{Li}_{2} \mathrm{IrO}_{3}$ as well as by studying the resonance behavior and the $\mathbf{q}$ dependence of the RIXS intensity. We find distinctive fingerprints of proximate Kitaev spin-liquid behavior: (i) a broad continuum of quasi-2D magnetic excitations centered around the $\Gamma$ point of the first Brillouin zone, (ii) the insensitivity of this continuum to the magnetic-ordering temperature $T_{N}$ and the persistence of the continuum up to temperatures as high as $300 \mathrm{~K}$, i.e., far above $T_{N}$, and (iii) dynamical spin-spin correlations restricted to nearest-neighbor bonds. The latter is a key feature of the Kitaev model on tricoordinated lattices. It explains the unusual temperature dependence of the RIXS intensity which, however, agrees with the behavior of the nearest-neighbor spin-spin correlations in the Kitaev model. Observations (i)-(iii) are comparable to the neutron results for $\alpha-\mathrm{RuCl}_{3}$ [17] discussed above. Under the additional consideration of dominant Kitaev interactions in the honeycomb iridates, our results for the magnetic excitations, in particular the combination of observations (i)-(iii), provide strong evidence for a proximate Kitaev spin liquid and establish a common experimental ground for these $j=1 / 2$ honeycomb compounds. Going beyond the thermodynamic perspective of the phase diagram sketched in Fig. 1, the magnetic continuum for excitation energies $\hbar \omega \gtrsim k_{B} T_{N}$ shows fingerprints of proximate Kitaev spin-liquid behavior even below the magnetic-ordering temperature-a spectroscopic proximate spin-liquid regime.

\section{CRYSTAL SYNTHESIS AND RIXS MEASUREMENTS}

Single crystals of $\mathrm{Na}_{2} \mathrm{IrO}_{3}$ can be grown by solid-state synthesis out of a polycrystalline bed [34]. Single crystals of several mm size were grown from $\mathrm{Na}_{2} \mathrm{CO}_{3}$ and Ir in a ratio of $1.5 / 1$ at $1423 \mathrm{~K}$. After cooling to room temperature the crystals were separated mechanically from the remaining polycrystalline sintered compact. Submillimeter single crystals of $\alpha-\mathrm{Li}_{2} \mathrm{IrO}_{3}$ can be grown from separated educts [35]. $\mathrm{Na}_{2} \mathrm{IrO}_{3}$ and $\alpha-\mathrm{Li}_{2} \mathrm{IrO}_{3}$ both crystallize in the monoclinic $C 2 / m$ space group [24], display opposite magnetic anisotropy, and order antiferromagnetically in zigzag and spiral states, respectively, near $15 \mathrm{~K}$ [13]. Crystal quality for the present study was checked by XRD and magnetic measurements. We use the reciprocal lattice of the monoclinic setting. In this 
framework, $\Gamma$ points of the 2D honeycomb net are given by, e.g., (0 0), (1 1 1), or (2 0$)$.

RIXS was measured at the Ir $L_{3}$ edge at beamline ID20 at the European Synchrotron Radiation Facility. The measurements were performed with incident $\pi$ polarization in the horizontal scattering plane in order to suppress the elastic line for $2 \theta$ close to $90^{\circ}$. The energy resolution equals $\Delta E=$ $24 \mathrm{meV}$, while the resolution of the transferred momentum q amounts to about $1 / 5$ of the Brillouin zone. The incident energy was tuned to the Ir $2 p_{3 / 2} \rightarrow 5 d_{t_{2 \Omega}}$ absorption edge, i.e., to resonate with the $t_{2 g}$ manifold forming the $j=1 / 2$ state. For both compounds, the measurements were performed on a (001) surface. The samples were oriented such that the $b$ axis was in the scattering plane and the $a$ axis perpendicular to it.

\section{EXPERIMENTAL RESULTS}

\section{A. Broad continuum of excitations}

One general challenge for RIXS studies of magnetic excitations in the honeycomb iridates is that the relevant excitation energies one has to cope with are comparably low. Therefore, we first address how, in our RIXS measurements, we distinguish inelastic features from the elastic line. Figure 2 shows exemplary low-energy RIXS spectra for $\alpha-\mathrm{Li}_{2} \mathrm{IrO}_{3}$ [(a) and (b)] and $\mathrm{Na}_{2} \mathrm{IrO}_{3}$ [(c) and (d)] at $5 \mathrm{~K}$ [(a) and (c)] and $300 \mathrm{~K}[(\mathrm{~b})$ and (d)]. The elastic line at zero energy loss with a width of $\Delta E=24 \mathrm{meV}$ overlaps with inelastic features that extend up to about $100 \mathrm{meV}$. Below we will show that this inelastic contribution corresponds to magnetic excitations. To disentangle inelastic features and the elastic line, we change

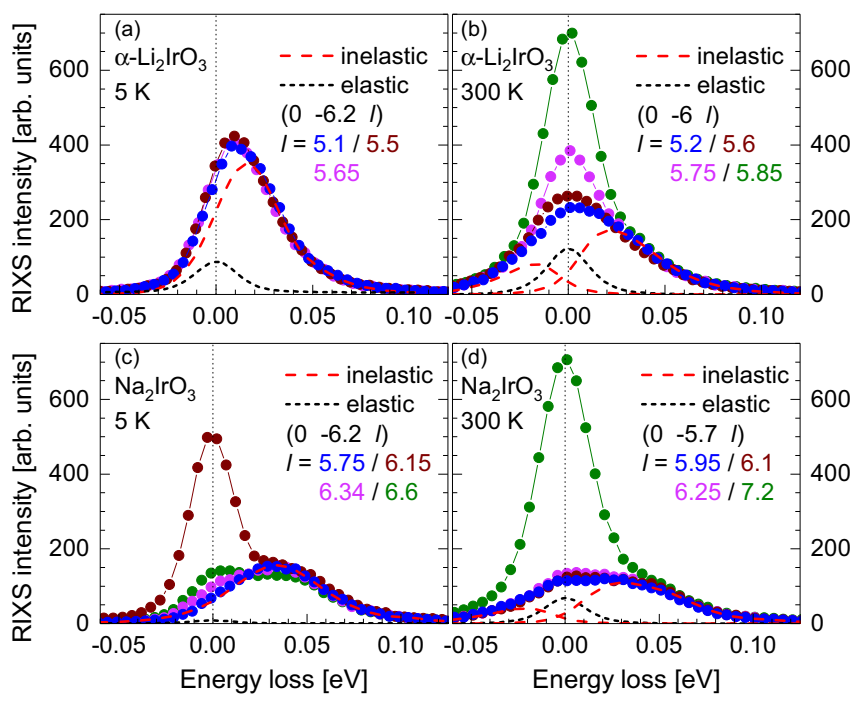

FIG. 2. Disentangling inelastic features from the elastic line. Low-energy RIXS spectra of $\alpha-\mathrm{Li}_{2} \mathrm{IrO}_{3}$ [(a) and (b)] and $\mathrm{Na}_{2} \mathrm{IrO}_{3}$ [(c) and (d)] at $5 \mathrm{~K}$ [(a) and (c)] and $300 \mathrm{~K}[(\mathrm{~b})$ and (d)] in the vicinity of $\mathbf{q}=(0-6 \mathrm{l})$. The width of the elastic line is $\Delta E=24 \mathrm{meV}$. Its intensity increases close to a Bragg peak and additionally depends on the scattering angle $2 \theta$. In particular, the elastic line is strongly suppressed for $2 \theta=90^{\circ}$. For the selected values of $l, 2 \theta$ covers the range from $90^{\circ}$ to $110^{\circ}$. The data above about $20 \mathrm{meV}$ are nearly independent of $l$, demonstrating the presence of inelastic features with a quasi-2D character.

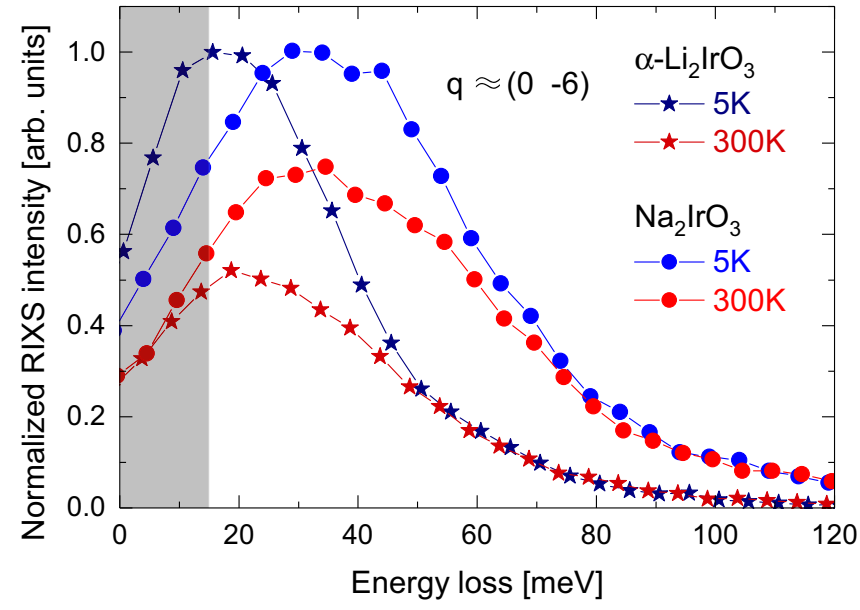

FIG. 3. Stokes contribution of RIXS spectra in the vicinity of $(h k)=(0-6)$. The data depict the result of the analysis shown in Fig. 2 and were normalized to the maximum value observed at $5 \mathrm{~K}$. We find a broad continuum which persists up to high temperature. Below $15 \mathrm{meV}$ (gray), the uncertainty increases since the precise intensity of the elastic line can only be estimated.

the intensity of the latter by varying the scattering angle $2 \theta$ in the range close to $90^{\circ}$. This is achieved by varying $l$ in the transferred momentum $\mathbf{q}=(h k l)$. Ideally, the elastic line is fully suppressed for $2 \theta=90^{\circ}$. We fit the spectra for different $l$ by a sum of elastic line (black dashed) and inelastic features (red dashed) using the Pearson VII line shape [36]. For the elastic line, this line shape and the width are determined by measurements on adhesive tape which do not show any inelastic contribution. At $300 \mathrm{~K}$, the fit includes an anti-Stokes contribution at negative energy loss, where the anti-Stokesto-Stokes intensity ratio is given by $\exp \left(-\hbar \omega / k_{B} T\right)$. In the fit, only the intensity of the elastic line is allowed to vary with $l$. The insensitivity to $l$ of the inelastic features reflects the quasi-2D character of the excitations.

The resulting Stokes contribution for transferred in-plane momenta close to $(0-6)$ is depicted in Fig. 3. Note that $(0-6)$ is equivalent to a $\Gamma$ point of the reciprocal lattice of the honeycomb net. At $5 \mathrm{~K}$, we find a broad excitation continuum peaking at about $15 \mathrm{meV}$ in $\alpha-\mathrm{Li}_{2} \mathrm{IrO}_{3}$ and $35 \mathrm{meV}$ in $\mathrm{Na}_{2} \mathrm{IrO}_{3}$. A continuum character is suggested by the large width of more than $50 \mathrm{meV}$, which is much larger than the experimental resolution $\Delta E=24 \mathrm{meV}$, and by the featureless line shape. A continuum reflects a two-particle or multiparticle character of the excitation, a hallmark of fractional excitations.

\section{B. Temperature dependence}

At $300 \mathrm{~K}$, the RIXS data are strikingly similar to the $5 \mathrm{~K}$ result, the main change being a reduction of the peak intensity; see Fig. 3. The temperature-driven reduction is larger in $\alpha-\mathrm{Li}_{2} \mathrm{IrO}_{3}$, in agreement with the smaller energy scale. For $\mathrm{Na}_{2} \mathrm{IrO}_{3}$, the striking robustness with respect to temperature is emphasized in Fig. 4 which shows that the RIXS peak for $q=\left(\begin{array}{lll}0 & 0 & 6.67\end{array}\right)$ hardly changes between $5 \mathrm{~K}$ and $90 \mathrm{~K} \approx 6 T_{N}$.

The insensitivity to temperature across $T_{N}=15 \mathrm{~K}$ and the robustness up to $6 T_{N}$ shows that the RIXS continuum cannot 


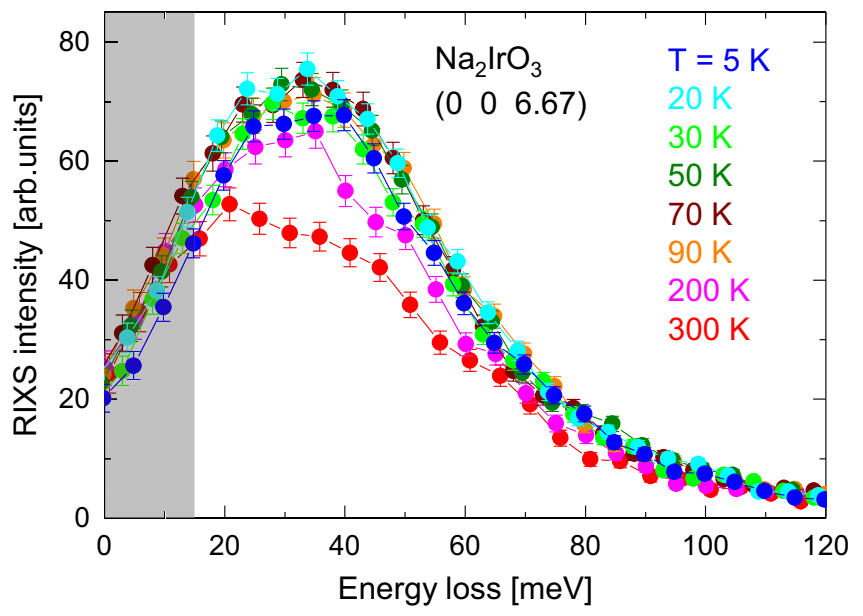

FIG. 4. Temperature dependence of the continuum in $\mathrm{Na}_{2} \mathrm{IrO}_{3}$. The plot depicts the Stokes contribution at $\left(\begin{array}{lll}0 & 0 & 6.67\end{array}\right)$. Data were normalized by the integrated RIXS intensity from 0.3 to $1.1 \mathrm{eV}$. The continuum hardly changes between $5 \mathrm{~K}$ and $90 \mathrm{~K}$ and persists up to $300 \mathrm{~K}$. In particular, the continuum is insensitive to the Néel temperature $T_{N}=15 \mathrm{~K}$. Below $15 \mathrm{meV}$ (gray), the uncertainty increases; cf. Fig. 3. Error bars refer to the raw data before subtraction of the elastic line; they do not represent the larger uncertainty in the gray area below $15 \mathrm{meV}$.

be explained in terms of magnons of the magnetically longrange ordered state observed below $T_{N}$. Also the quasi-2D character speaks against magnons of the 3D ordered state. As discussed in the next section, our RIXS data nevertheless provide strong evidence for a magnetic character of the continuum, pointing toward a more intriguing origin. A dominant Kitaev exchange is well accepted for the honeycomb iridates [11-13], in which long-range order is thought to arise due to additional weaker coupling terms beyond the pure Kitaev model [12-14]. This suggests an analogy with quasi-one-dimensional systems which show long-range order at low temperature due to finite interchain couplings. Nevertheless the excitations at higher energy are well described as fractional spinon excitations, both below and above the ordering temperature. For the iridates, this suggests that the proximate Kitaev spin liquid leaves its fingerprints not only in the magnetic excitations above the ordering temperature, as sketched in Fig. 1, but also in the high-energy magnetic excitations for temperatures below the long-range ordering temperature. In this scenario, long-range order mainly affects the excitations at lower energy, i.e., below $20 \mathrm{meV}$ in $\mathrm{Na}_{2} \mathrm{IrO}_{3}$. The high-energy Kitaev-related excitations are much more robust against temperature than conventional magnons.

\section{Magnetic character and resonance behavior}

At first sight, a magnetic interpretation of excitations that show such low sensitivity to temperature is counterintuitive and requires a solid experimental foundation, which we will give in the following. Our RIXS spectra of $\mathrm{Na}_{2} \mathrm{IrO}_{3}$ shown in Fig. 4 agree very well with the data reported by Gretarsson et al. [33]. They interpreted their data in terms of a series of overlapping phonons at high temperature and as a sum of phonons and magnons at low temperature [33]. In their scenario, the small temperature dependence of the intensity on the Stokes side is due to an accidental balance of the phonon intensity increasing and the magnon intensity decreasing with increasing temperature. A first argument against this scenario is provided by the comparison of the data for isostructural $\alpha-\mathrm{Li}_{2} \mathrm{IrO}_{3}$ and $\mathrm{Na}_{2} \mathrm{IrO}_{3}$; see Fig. 3. Phonons are expected to be very similar in these two compounds, with an enhanced energy scale in $\alpha-\mathrm{Li}_{2} \mathrm{IrO}_{3}$ due to the smaller mass of $\mathrm{Li}$ compared to $\mathrm{Na}$, as observed in optical spectroscopy [37]. In contrast, the energy scale is markedly lower in $\alpha-\mathrm{Li}_{2} \mathrm{IrO}_{3}$ in the RIXS data, questioning the phonon interpretation.

In RIXS at the Ir $L_{3}$ edge, magnetic excitations can be distinguished from phonons by their resonance behavior. Magnetic spin-flip excitations and orbital excitations are created in a direct RIXS process. Starting from a local $j=1 / 2$ ground state with a $2 p^{6} 5 d^{5}$ configuration in which the five $5 d$ electrons occupy the $t_{2 g}$ shell, the incident photon energy of $11.2145 \mathrm{keV}$ is tuned to resonantly enhance the absorption process that promotes a $2 p$ core electron to the $5 d t_{2 g}$ level, resulting in a $2 p^{5} t_{2 g}^{6}$ intermediate state. Within a few femtoseconds, this intermediate state relaxes to the final state by photon emission, resulting in an excited $2 p^{6} t_{2 g}^{5 *}$ state that corresponds to a magnetic or orbital excitation. The resonance strongly boosts the RIXS intensity of these excitations. In contrast, a phonon excitation is an indirect RIXS process which arises due to the dynamics in the short-lived intermediate state; i.e., the lattice distorts to screen the core hole before the electronic system relaxes from the intermediate state to the $j=1 / 2$ ground state. Typically, the cross section for direct RIXS is much larger [38]. Phonons are expected to be particularly weak in RIXS if the core hole in the intermediate state is well screened [38], as commonly assumed for the Ir $2 p^{5} t_{2 g}^{6}$ intermediate state with localized $t_{2 g}$ states. This explains why no detectable phonon contribution was observed thus far in $L$-edge RIXS data of other Mott-insulating $5 d^{5}$ iridates [26-30,33,39-45]. A significant phonon contribution requires a more delocalized intermediate state, in which case the core hole is screened by adjacent ions and the lattice distorts. Since the $e_{g}$ orbitals are both more delocalized and more strongly coupled to the lattice, the intensity of a hypothetical phonon peak is expected to be enhanced at the $e_{g}$ resonance [46], which occurs at about $E_{\text {in }}=11.218 \mathrm{keV}$ in the $5 d^{5}$ iridates [47]. The $3.5 \mathrm{eV}$ difference in resonance energy between $t_{2 g}$ and $e_{g}$ levels corresponds to the cubic crystal field splitting.

The resonance behavior thus provides a litmus test for the magnetic character of excitations. In the iridates, magnetic excitations and intra- $t_{2 g}$ orbital excitations both resonate at $E_{\text {in }}=11.2145 \mathrm{keV}$, whereas the RIXS intensity of phonons is expected to show a different resonance behavior. In Mottinsulating $\alpha-\mathrm{Li}_{2} \mathrm{IrO}_{3}$ and $\mathrm{Na}_{2} \mathrm{IrO}_{3}$, intra- $t_{2 g}$ excitations set in at $0.4 \mathrm{eV}$ with a peak at about $0.7 \mathrm{eV}$ for spin-orbital excitations to the $j=3 / 2$ state [41]; see Fig. 5. The resonance maps depicted in Fig. 6 show the same resonance behavior for the RIXS continuum below $0.1 \mathrm{eV}$ and the intra- $t_{2 g}$ excitations above $0.4 \mathrm{eV}$, both resonating at $E_{\text {in }}=11.2145 \mathrm{keV}$. We emphasize that an $e_{g}$ resonance at $11.218 \mathrm{keV}$ is not observed.

The experimental result that all excitations in the measured energy range show the same resonance behavior is particularly obvious from cuts through the resonance maps. Normalized constant-loss cuts below $0.1 \mathrm{eV}$ and around 0.6 to $0.8 \mathrm{eV}$ agree 


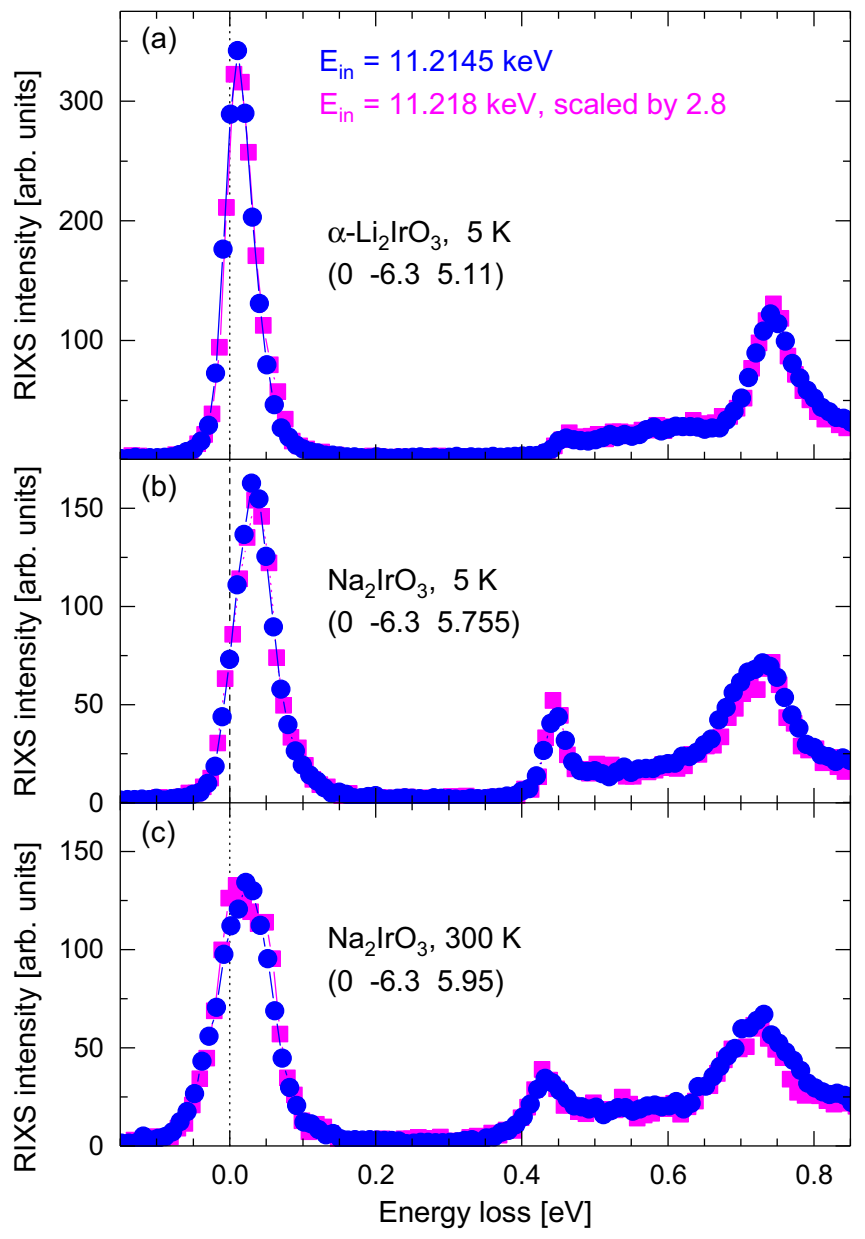

FIG. 5. Resonance behavior of RIXS spectra, establishing the magnetic character of the low-energy continuum. RIXS was measured on $\alpha-\mathrm{Li}_{2} \mathrm{IrO}_{3}$ (a) and $\mathrm{Na}_{2} \mathrm{IrO}_{3}$ [(b) and (c)] for incident energies of $11.2145 \mathrm{keV}$ and $11.218 \mathrm{keV}$, equivalent to constant- $E_{\text {in }}$ cuts through the resonance maps shown in Fig. 6. To suppress the elastic line, the transferred momentum was chosen such that $2 \theta \approx 90^{\circ}$. Maximum resonance enhancement is obtained at the $t_{2 g}$ resonance with $E_{\text {in }}=11.2145 \mathrm{keV}$; see Fig. 7. Data for $E_{\text {in }}=11.218 \mathrm{keV}$ were scaled up by a factor 2.8 to compensate for detuning $E_{\text {in }}$ away from the $t_{2 g}$ resonance. After scaling, the spectra fall on top of each other over the full measurement range, demonstrating that the excitation occurs via a direct RIXS process for all observed features.

very well with each other, showing a maximum resonance enhancement at $11.2145 \mathrm{keV}$ independent of the energy loss; see Fig. 7. Cuts for constant $E_{\text {in }}=11.2145 \mathrm{keV}$ and $11.218 \mathrm{keV}$ are compared in Fig. 5. Also these spectra fall on top of each other after normalization. For the normalization, the data for $E_{\text {in }}=11.218 \mathrm{keV}$ were scaled up by a factor of 2.8 , which compensates the detuning of $E_{\text {in }}$ away from the $t_{2 g}$ resonance. This scaling behavior proves a common electronic intra- $t_{2 g}$ character of the entire excitation spectrum below $1 \mathrm{eV}$, where "electronic" refers to magnetic and orbital degrees of freedom at low and high energies, respectively. Most noteworthy, this still holds true at $300 \mathrm{~K}$; see Fig. 5(c). Thus, we conclude that a phonon interpretation of the low-energy continuum can be firmly ruled out.
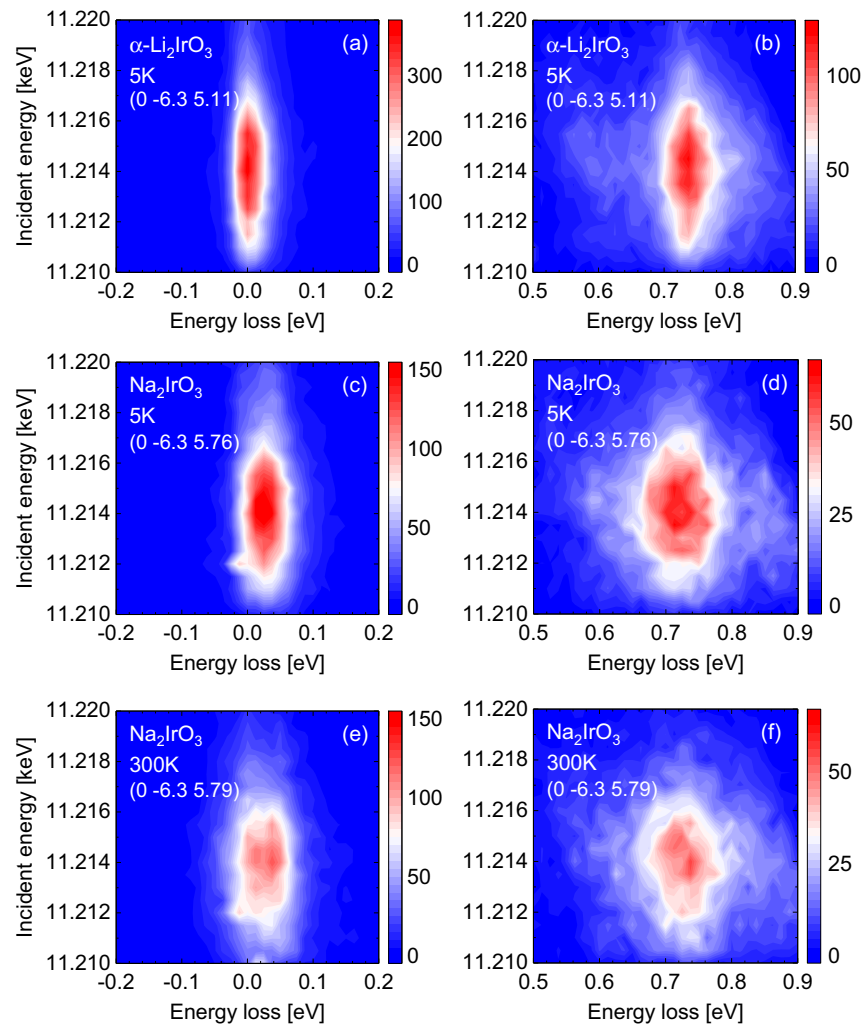

FIG. 6. Resonance maps. With RIXS being a resonant technique, the intensity strongly depends on the incident energy which here is varied across the $\mathrm{Ir} L_{3}$ edge. Data were measured for $\alpha-\mathrm{Li}_{2} \mathrm{IrO}_{3}$ at $5 \mathrm{~K}$ [(a) and (b)] and $\mathrm{Na}_{2} \mathrm{IrO}_{3}$ at $5 \mathrm{~K}$ [(c) and (d)] and $300 \mathrm{~K}$ [(e) and (f)]. (a), (c), (e): Low-energy magnetic excitations. (b), (d), (f): Intra- $t_{2 g}$ excitations to the $j=3 / 2$ state. All panels show a pronounced $t_{2 g}$ resonance at $E_{\text {in }}=11.2145 \mathrm{keV}$ but no resonance at the $e_{g}$ resonance energy $E_{\text {in }}=11.218 \mathrm{keV}$. The elastic line is suppressed by choosing $2 \theta \approx 90^{\circ}$. Cuts through the resonance maps are plotted in Figs. 5 and 7 .

\section{Nearest-neighbor spin-spin correlations}

Having established the magnetic character of the continuum, we now address the q-dependent behavior. RIXS spectra of $\mathrm{Na}_{2} \mathrm{IrO}_{3}$ covering the range from $(0-4.46 .5)$ to $(0-5.4$ $6.5)$ are depicted in Fig. 8. Note that $(0-56.5)$ corresponds to a magnetic Bragg peak [48]. The data in Fig. 8 were normalized to the RIXS intensity at an energy loss of $50 \mathrm{meV}$. The excellent agreement obtained above some $20 \mathrm{meV}$ shows that the RIXS intensity of the magnetic continuum depends on $\mathbf{q}$ while the peak energy and width are hardly affected. The q-dependent intensity of the continuum corresponds to the dynamical structure factor $S(\mathbf{q}, \omega)$, which allows us to measure dynamical spin-spin correlations in real space (see also the Discussion section).

We address $S(\mathbf{q}, \omega)$ by measuring the q-dependent intensity modulation of the magnetic continuum in the range of $30 \mathrm{meV}$ to $150 \mathrm{meV}$ for different directions of $\mathbf{q}$; see Fig. 9. The choice of $30 \mathrm{meV}$ for the cutoff is adapted to the experimental resolution. Note that the result is qualitatively not affected by choosing larger values for the cutoff. The data cover several Brillouin zones, both at $5 \mathrm{~K}$ and $300 \mathrm{~K}$. The honeycomb lattice has three different $\mathrm{Ir}-\mathrm{Ir}$ bonds which are 


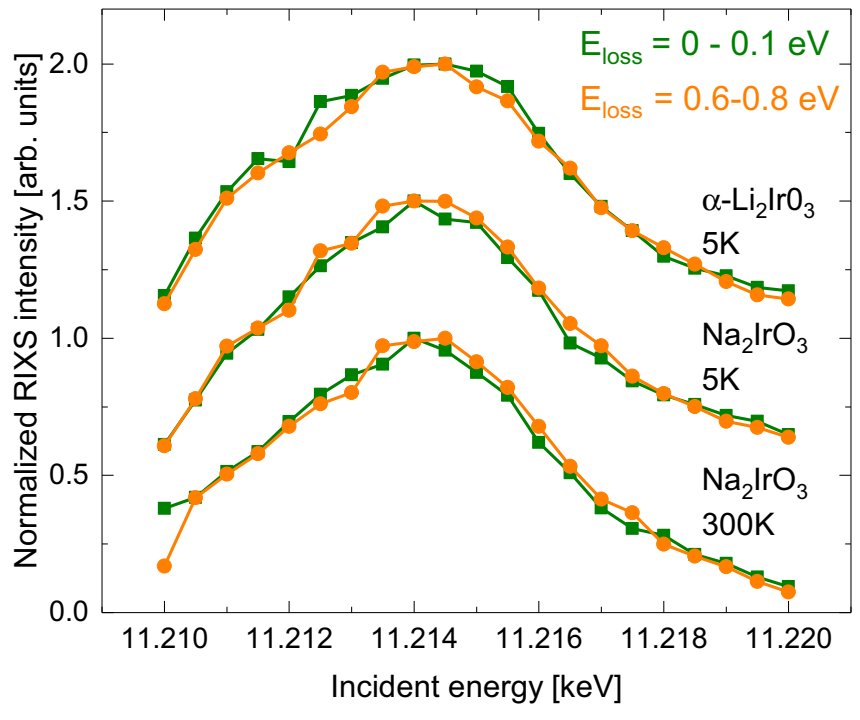

FIG. 7. Constant-loss cuts through the resonance maps shown in Fig. 6. Data were normalized to the maximum value and are offset for clarity. In both compounds, the low-energy excitations (green) show the same resonance behavior as the intra- $t_{2 g}$ excitations around $0.7 \mathrm{eV}$ (orange), exhibiting a pronounced resonance peak at $11.2145 \mathrm{keV}$. This demonstrates the direct RIXS character of the excitation process and establishes the magnetic character of the low-energy continuum, both at $5 \mathrm{~K}$ and at $300 \mathrm{~K}$.

described by the real-space vectors $\mathbf{r}_{1,3}=( \pm a / 2, b / 6,0)$ and $\mathbf{r}_{2}=(0, b / 3,0)$, as sketched by the green, red, and blue bonds of Fig. 9(a) and 9(b). In Fig. 9(c) and 9(e), we show data for $\mathbf{q} \perp \mathbf{r}_{1}$ with $\mathbf{q} \cdot \mathbf{r}_{2}=\mathbf{q} \cdot \mathbf{r}_{3}(\Gamma-K$ direction; cf. inset of Fig. 8). In this direction, we find a pronounced sinusoidal

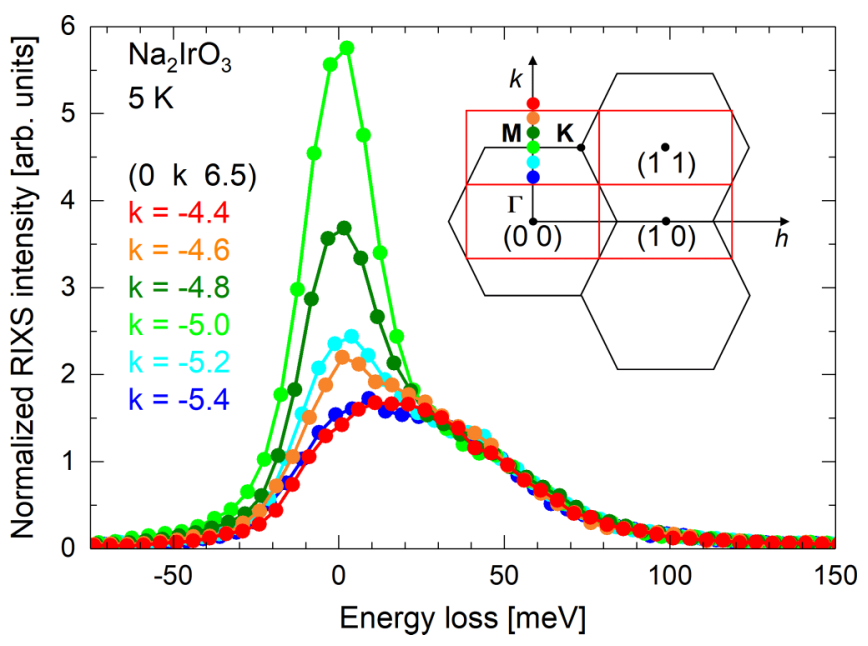

FIG. 8. Momentum dependence of normalized RIXS spectra of $\mathrm{Na}_{2} \mathrm{IrO}_{3}$. Data were normalized by the intensity at $50 \mathrm{meV}$ energy loss. Above about $20 \mathrm{meV}$, the normalized spectra fall on top of each other, showing that concerning the continuum, only the intensity changes as a function of $\mathbf{q}$. Inset: Black lines denote the 2D Brillouin zone of the honeycomb lattice with the high-symmetry points $\Gamma, K$, and $M$. Red rectangles depict 2D cuts of the Brillouin zone of the monoclinic lattice. The colors of the symbols denote the $\mathbf{q}$ values equivalent to the ones studied in the main figure. intensity modulation $\cos ^{2}\left(\mathbf{q} \cdot \mathbf{r}_{2} / 2\right)$ in both compounds. This behavior strongly deviates from the expectations for magnons in a long-range ordered state, as reported in $L$-edge RIXS on the square-lattice Heisenberg magnet $\mathrm{Sr}_{2} \mathrm{IrO}_{4}$ [26]. Such a sinusoidal modulation, however, fully agrees with the Kitaev model for which one finds [17]

$$
S(\mathbf{q}, \omega) \propto \sum_{i}\left[S_{0}(\omega)-K_{1} S_{1}(\omega)+2 K_{1} S_{1}(\omega) \cos ^{2}\left(\frac{\mathbf{q} \cdot \mathbf{r}_{i}}{2}\right)\right]
$$

for ferromagnetic Kitaev coupling $K_{1}$, where $S_{0}$ and $S_{1}$ denote on-site and nearest-neighbor correlators, respectively. Recently, such a sinusoidal modulation of the Ir $L$-edge RIXS intensity was observed for Ir dimers in $\mathrm{Ba}_{3} \mathrm{CeIr}_{2} \mathrm{O}_{9}$ where it was discussed in terms of RIXS interferometry and interpreted as an inelastic incarnation of Young's double-slit experiment [44]. For the dimers, the superposition of x-ray photons scattered coherently on the two sites yields the prototypical sinusoidal interference pattern of two-beam interference. Applied to the magnetic continuum, our data show a modulation period which corresponds to nearest-neighbor correlations without any evidence for a shorter modulation period; see Fig. 9(c) and 9(e). This shows that a given excited state can only be reached by spin-flip RIXS processes on either of the two Ir sites of a given bond, which is equivalent to a double-slit-type process. In other words, dynamical spin-spin correlations for the magnetic continuum are restricted to nearest neighbors; we find no signature of correlations between further neighbors (see below). This is a key feature of the Kitaev model, in which the three different spin-flip channels are independent and do not interfere with each other [50]. Flipping the $x$ component of the spin creates an excitation on an $x$ bond, while a flip of the $y$ component yields an orthogonal final state living on a $y$ bond. Similar results were reported for $\alpha-\mathrm{RuCl}_{3}$ based on inelastic neutron scattering [17]. Remarkably, $\alpha-\mathrm{Li}_{2} \mathrm{IrO}_{3}$ and $\mathrm{Na}_{2} \mathrm{IrO}_{3}$ show very similar behavior, despite their different magnetic ordering patterns [13]. This further supports the notion that the restriction to nearest-neighbor correlations is due to dominant Kitaev exchange and insensitive to small additional couplings.

Besides the sinusoidal modulation, the RIXS intensity shows a slow variation with $\mathbf{q}$. Note that a change of $\mathbf{q}$ is unavoidably accompanied by a change of the experimental scattering geometry, which affects the x-ray polarization and the matrix elements. This gives rise to deviations from a pure sinusoidal behavior. We emphasize that spin-spin correlations beyond nearest neighbors would give rise to a shorter modulation period, for which we do not find any evidence for $\hbar \omega \geqslant 30 \mathrm{meV}$. More explicitly, the scattering geometry chosen for the data in Figs. 9(c) and 9(e) yields a RIXS intensity

$$
I_{\perp}(\mathbf{q}) \propto I_{0}+I_{2,3} \cos ^{2}\left(\mathbf{q} \cdot \mathbf{r}_{2,3} / 2\right)+I_{\mathrm{fn}} \cos ^{2}\left(\mathbf{q} \cdot \mathbf{r}_{2}\right),
$$

where the term proportional to $I_{2,3}$ refers to nearest neighbors at $\mathbf{r}_{2}$ and $\mathbf{r}_{3}$. For more distant neighbors, $\mathbf{q} \cdot \mathbf{r}_{i}$ in this geometry with $\mathbf{q} \perp \mathbf{r}_{1}$ is an integer multiple of $\mathbf{q} \cdot \mathbf{r}_{2}$, and Eq. (2) considers only the contribution with the largest modulation period, which, beyond next-nearest neighbors, also contains contributions from third-nearest and more distant neighbors. A least-squares fit of the $5 \mathrm{~K}$ data of $\alpha-\mathrm{Li}_{2} \mathrm{IrO}_{3}\left(\mathrm{Na}_{2} \mathrm{IrO}_{3}\right)$ 
(a)

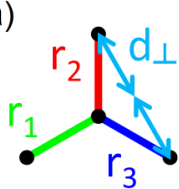

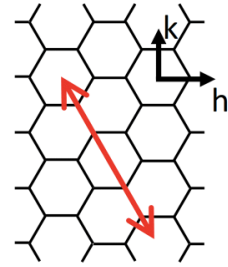

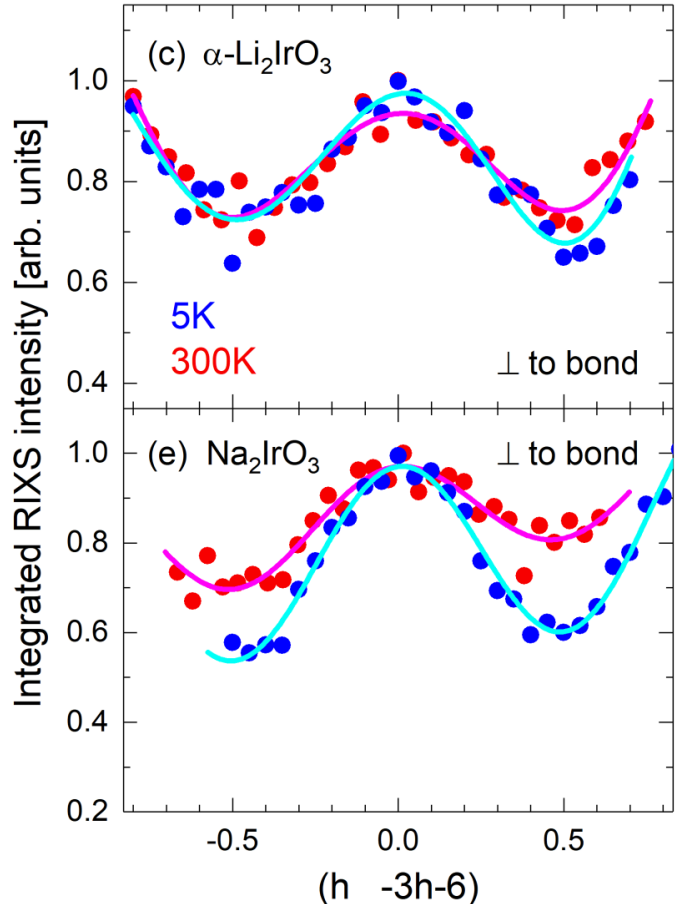

(b)
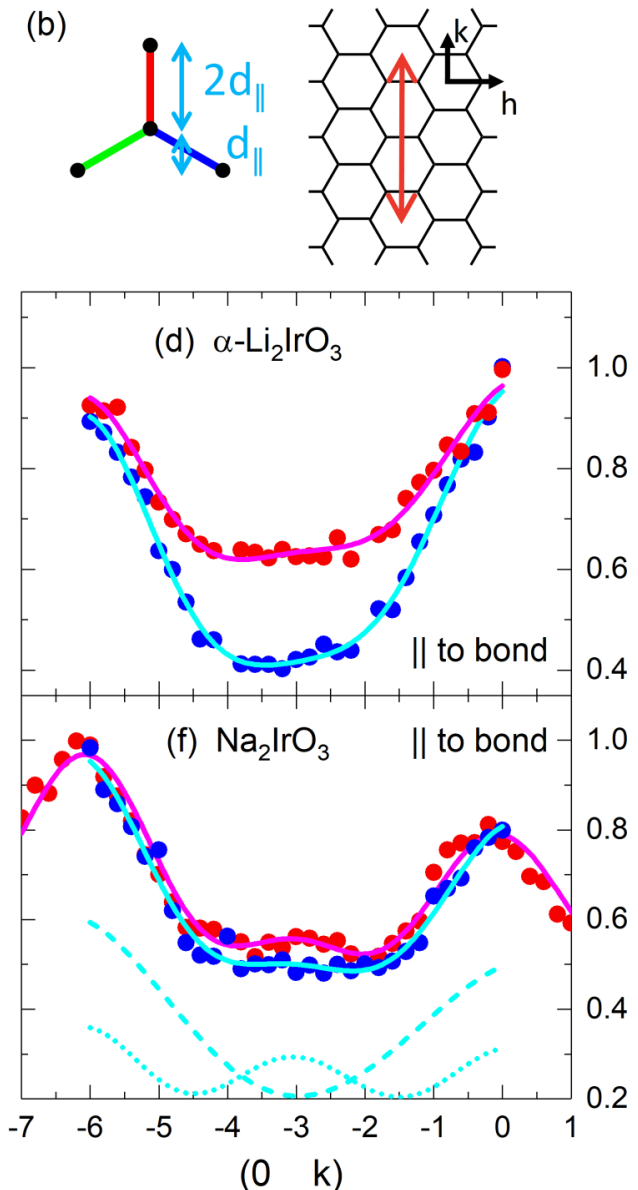

FIG. 9. RIXS interference patterns showing only nearest-neighbor spin-spin correlations. (a): Sketches of the three Ir-Ir bonds $\mathbf{r}_{i}$ (green, red, and blue) and of several Brillouin zones of the reciprocal lattice (black). The red arrow marks the range of $\mathbf{q}$ covered by the data in (c) and (e) along $\Gamma-K$. Light blue arrows depict the projection of $\mathbf{r}_{i}$ on q. (b): Same for the $\Gamma-M$ direction. (c)-(f): Normalized RIXS intensity as a function of $\mathbf{q}$, integrated from 30 to $150 \mathrm{meV}$. Data were corrected for self-absorption effects [49]. Solid lines: Sinusoidal fits assuming that dynamical spin-spin correlations are restricted to nearest neighbors. (c), (d): $\alpha-\mathrm{Li}_{2} \mathrm{IrO}_{3}$. (e), (f): $\mathrm{Na}_{2} \mathrm{IrO} \mathrm{O}_{3}$. (c) and (e): Data for $\mathbf{q}$ along $\Gamma-K$, as sketched in (a). Along $\Gamma-K$, the projection of $\mathbf{q}$ on the Ir-Ir bonds yields a single sinusoidal modulation. (d) and (f): Data for $\mathbf{q}$ along $\Gamma-M$, as sketched in (b). Along $\Gamma-M$, nearest-neighbor correlations along the three different bonds yield a superposition of two modulation periods, see Eq. (3), as observed experimentally. Dashed and dotted lines in (f) exemplarily show the two individual contributions to the fit at 5 K. The interference patterns are remarkably robust against temperature.

yields $I_{\mathrm{fn}} / I_{2,3} \approx 0.03 \pm 0.15(0.02 \pm 0.07)$, suggesting a vanishing contribution of further neighbors. Setting $I_{\mathrm{fn}}=0$ has a marginal effect on the fit quality, with $\chi^{2}$ increasing by less than $1 \%$. Based on the noise level of the data, we conservatively estimate the upper limit for such a furtherneighbor contribution with a shorter modulation period to $10 \%$.

Quantitatively, the modulation amplitude contains important information on the ratio $S_{1} / S_{0}$; see Eq. (1). Such a quantitative analysis, however, requires detailed measurements of, e.g., the polarization dependence and is beyond the scope of our present work. In the neutron study of $\alpha-\mathrm{RuCl}_{3}$, $S_{1} / S_{0}$ was scaled by a phenomenological prefactor that describes deviations from the pure Kitaev model.

The restriction of dynamical spin-spin correlations to nearest neighbors is corroborated by the behavior observed for the $\Gamma-M$ direction; see Figs. 9(d) and 9(f). With $\mathbf{q} \| \mathbf{r}_{2}$ and $\mathbf{q} \cdot \mathbf{r}_{1}=\mathbf{q} \cdot \mathbf{r}_{3}=\left(\mathbf{q} \cdot \mathbf{r}_{2}\right) / 2$, the nearest-neighbor correlations on the three different bonds give rise to an incoherent sum of two sinusoidal modulations,

$$
I(\mathbf{q}) \propto I_{0}+I_{2} \cos ^{2}\left(\mathbf{q} \cdot \mathbf{r}_{2} / 2\right)+I_{1,3} \cos ^{2}\left(\mathbf{q} \cdot \mathbf{r}_{1,3} / 2\right),
$$

where the period of the latter is twice the period of the former due to the different projections $\mathbf{q} \cdot \mathbf{r}_{i}$; see dashed and dotted lines in Fig. 9(f). For instance for $\mathrm{Na}_{2} \mathrm{IrO}_{3}$, the experiment roughly shows $I_{1,3} \approx 2.4 I_{2}$, which reflects the fact that the two bonds $\mathbf{r}_{1}$ and $\mathbf{r}_{3}$ contribute to the modulation amplitude $I_{1,3}$. Additionally, the prefactors depend on the $\mathrm{x}$-ray polarization and the scattering geometry, both changing with $\mathbf{q}$ in our measurements. As discussed above for the $\Gamma-K$ direction, this causes deviations from a pure sinusoidal behavior.

Most importantly, we do not find, within our experimental resolution and for $\hbar \omega \geqslant 30 \mathrm{meV}$, any evidence for further modulation periods beyond nearest neighbors. Taking into account a term $I_{\mathrm{fn}} \cos ^{2}\left(3 \mathbf{q} \cdot \mathbf{r}_{1} / 2\right)$ which represents four bonds to next-nearest neighbors as well as further neighbors again does not increase the fit quality substantially, as discussed above for $\mathbf{q} \perp \mathbf{r}_{1}$. For the $\Gamma$ - $M$ direction with $\mathbf{q} \| \mathbf{r}_{2}$ 


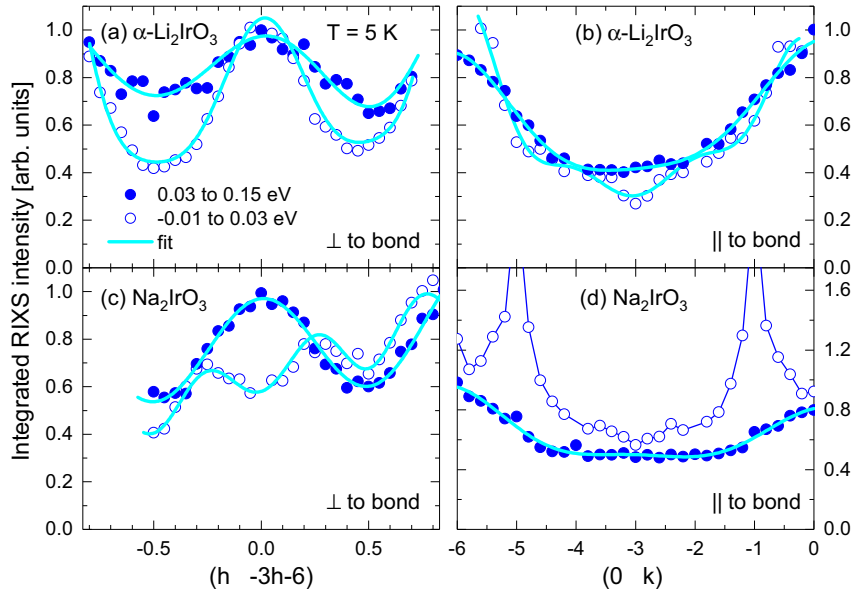

FIG. 10. RIXS intensity in the long-range magnetically ordered state at 5 K. Full symbols: Same data as in Fig. 9, showing the normalized RIXS intensity integrated from 30 to $150 \mathrm{meV}$. Open symbols show corresponding low-energy data integrated from -10 to $+30 \mathrm{meV}$, revealing differences between the two compounds that can be attributed to the different magnetic ordering patterns. In contrast, the behavior at high energy (full symbols) is identical. (a), (b): $\alpha-\mathrm{Li}_{2} \mathrm{IrO}_{3}$. (c), (d): $\mathrm{Na}_{2} \mathrm{IrO}_{3}$. (a) and (c): $\Gamma-K$ direction (perpendicular to an Ir-Ir bond; see Fig. 9). (b) and (d): $\Gamma-M$ direction (parallel to an Ir-Ir bond). Data were corrected for self-absorption effects [49].

we find $I_{\mathrm{fn}} / I_{1,3}=0.03 \pm 0.06(0.07 \pm 0.08)$ for $\alpha-\mathrm{Li}_{2} \mathrm{IrO}_{3}$ $\left(\mathrm{Na}_{2} \mathrm{IrO}_{3}\right)$, with $\chi^{2}$ decreasing by $3 \%(14 \%)$. This supports our estimate for an upper limit of further-neighbor contributions of $10 \%$.

The q-dependent intensity modulation is very robust against temperature. The main change at $300 \mathrm{~K}$ is a reduction of the modulation amplitude; see Fig. 9. This can be rationalized by the energy scale of the observed excitations, which is comparable to $k_{B} 300 \mathrm{~K} \approx 26 \mathrm{meV}$. It is thus not surprising that nearest-neighbor correlations are still strong at $300 \mathrm{~K}$.

Our central experimental finding is that the magnetic continuum shows only nearest-neighbor spin-spin correlations even in the long-range magnetically ordered state at $5 \mathrm{~K}$. This behavior strongly deviates from the expectations for magnons, which are supposed to reflect longer-range spin-spin correlations at lower energy. Indeed, for integration below $30 \mathrm{meV}$ at $5 \mathrm{~K}$, both $\mathrm{Na}_{2} \mathrm{IrO}_{3}$ and $\alpha-\mathrm{Li}_{2} \mathrm{IrO}_{3}$ show additional shorter modulation periods which correspond to spin-spin correlations beyond nearest neighbors; see open symbols in Fig. 10. Note that the magnetic ordering patterns differ in the two compounds. Zigzag order in $\mathrm{Na}_{2} \mathrm{IrO}_{3}$ gives rise to a magnetic Bragg peak at, e.g., $(0-16.5)$, which explains the strong increase of intensity in Fig. 10(d). In contrast, $\alpha-\mathrm{Li}_{2} \mathrm{IrO}_{3}$ exhibits incommensurate magnetic order with the ordering wave vector $( \pm 0.3200)$. The different magnetic ordering patterns explain the differences in the dynamical structure factors $S(\mathbf{q}, \omega)$ of the two compounds at low energy for temperatures below $T_{N}$. These differences observed at low energy corroborate the notion that the common behavior of the highenergy continuum is not related to long-range magnetic order, as sketched in Fig. 1.


(b)

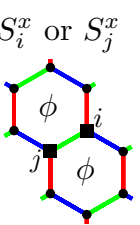

FIG. 11. Flux excitations in the Kitaev model. In the Kitaev model, spin-flip RIXS on site $i$ creates two flux excitations, so-called visons, on adjacent plaquettes. (a) For excitation on site $i$, there are three orthogonal excited states; each corresponds to a different spinflip channel $S_{i}^{\alpha}$ [50]. (b) Each excited state can be reached only from the two sites $i$ and $j$ on the bond connecting the respective plaquettes. This restricts dynamical spin-spin correlations to nearest-neighbor sites and explains the sinusoidal RIXS interference patterns depicted in Fig. 9.

\section{DISCUSSION}

Our RIXS study of $\mathrm{Na}_{2} \mathrm{IrO}_{3}$ and $\alpha-\mathrm{Li}_{2} \mathrm{IrO}_{3}$ in the hightemperature regime above their respective magnetic ordering transitions as well as the high-energy regime of their magnetically ordered low-temperature states has revealed many atypical features that allow us to clearly rule out that this high-energy and high-temperature RIXS response can be interpreted as arising from a conventional, featureless paramagnetic state. In fact, taken together these experimentally observed features-a broad continuum of magnetic excitations, its persistence to high temperatures, and dominant nearest-neighbor spin-spin correlations-provide strong evidence, as we will argue in the following, for the notion that $\mathrm{Na}_{2} \mathrm{IrO}_{3}$ and $\alpha-\mathrm{Li}_{2} \mathrm{IrO}_{3}$ are both in close proximity to a Kitaev spin liquid. We will review the RIXS response expected for the pure Kitaev model and discuss how this response is modified in the presence of additional magnetic interactions [51], i.e., generic diagonal and off-diagonal couplings expected to be present in these spin-orbit entangled materials [12].

RIXS response of pure Kitaev model. For the pure Kitaev model, the RIXS response was first elucidated by Halász, Perkins, and van den Brink [50], where they showed that, with a $t_{2 g}^{6}$ intermediate state, the response naturally decomposes into four independent RIXS channels. The single spin-conserving RIXS channel corresponds to an indirect RIXS process and is thus much weaker than the remaining three spin-flip RIXS channels, which correspond to direct processes. As a result, it is only these spin-flip RIXS channels that are of relevance to the discussion here. The three different channels correspond to the three diagonal components of the dynamical spin structure factor. In the language of the Kitaev model, they excite both mobile Majorana fermions and static flux excitations, so-called visons. For example, the operator $S_{i}^{x}$ creates propagating Majoranas, as well as two visons in adjacent plaquettes, as illustrated in Fig. 11(a). Since the visons are gapped, this translates to a gapped RIXS response, with a sharp peak at the $\Gamma$ point at the two-flux gap of the order of $10 \%$ of the Kitaev coupling $K_{1}$, followed by a broad continuиm, reflecting the existence of fractionalized excitations (itinerant Majorana fermions and static visons).

Broad continuum of excitations. Experimentally, the total spin-flip RIXS spectrum shows only a broad magnetic 
excitation continuum at the $\Gamma$ point, peaking at about $15 \mathrm{meV}$ in $\alpha-\mathrm{Li}_{2} \mathrm{IrO}_{3}$ and $35 \mathrm{meV}$ in $\mathrm{Na}_{2} \mathrm{IrO}_{3}$. The expectation of the pure Kitaev model, a dominant sharp peak at $\hbar \omega \sim 0.1\left|K_{1}\right|$ followed by a broad continuum, must be modified to include (i) the anisotropy of the Kitaev coupling on different bonds [51], (ii) the inclusion of additional exchange interactions such as Heisenberg and off-diagonal, bond-directional terms (such as the so-called $\Gamma$ exchange) [12], and (iii) finite temperature. Taken together, all three effects lead to a significant broadening of the dominant peak and a shift to higher energies [52-56]. For both $\mathrm{Na}_{2} \mathrm{IrO}_{3}$ and $\alpha-\mathrm{Li}_{2} \mathrm{IrO}_{3}$ the anisotropy of $K_{1}$ and the existence of additional exchange interactions are well established [51]. Unfortunately, inclusion of such terms breaks the integrability of the model, providing a challenge to quantitative theoretical modeling. However, an augmented parton mean-field theory from Knolle, Bhattacharjee, and Moessner [53] found that the dominant low-energy peak does indeed broaden substantially and shift to significantly higher energy upon inclusion of the additional relevant exchange interactions. Without detailed knowledge of the minimal model for the materials at hand, one can only estimate that the peak energy should be comparable to the energy scale of the Kitaev coupling. Experimental and theoretical results consistently show that the latter is larger in $\mathrm{Na}_{2} \mathrm{IrO}_{3}$ than in $\alpha-\mathrm{Li}_{2} \mathrm{IrO}_{3}$. Specific heat data [57] show that the peak temperature, identified with the upper crossover in Fig. 1, is about $20 \%$ higher in $\mathrm{Na}_{2} \mathrm{IrO}_{3}$. Ab initio estimates [51,58-60] yield ferromagnetic $K_{1}$ in the range -15 to $-35 \mathrm{meV}$ for $\mathrm{Na}_{2} \mathrm{IrO}_{3}$ and -4 to $-13 \mathrm{meV}$ for $\alpha-\mathrm{Li}_{2} \mathrm{IrO}_{3}$. This is in good agreement with our observation that the RIXS peak energy is roughly a factor of two higher in $\mathrm{Na}_{2} \mathrm{IrO}_{3}$ and that $\mathrm{Na}_{2} \mathrm{IrO}_{3}$ is less sensitive to temperature. The broad excitation continuum observed is thus consistent with the expectation for an extended Kitaev model.

Temperature dependence of continuum. For a magnetic excitation, the experimentally observed broad continuum displays a highly unusual insensitivity to temperature. The shape of the continuum remains largely unchanged with increasing temperature, and also the intensity is roughly constant up to about $6 T_{N}$; see Fig. 4 . Even up to room temperature, we find only a moderate decrease in overall intensity, which is more pronounced for $\alpha-\mathrm{Li}_{2} \mathrm{IrO}_{3}$, see Fig. 3, consistent with the overall lower energy scale of the peak. Qualitatively, this remarkable temperature dependence is consistent with numerical calculations, where, at temperatures above the flux gap, the broad continuum displays only a smooth weakening in intensity as temperature increases [54,56,61,62]. For a quantitative analysis, we make use of our finding that the magnetic continuum reveals only nearest-neighbor correlations; see Fig. 9. This motivates us to compare the integrated RIXS intensity of the continuum with the (static) nearestneighbor correlations of the pure Kitaev model, calculated through numerically exact quantum Monte Carlo simulations [63]. Note that we consider static correlations in our numerics, i.e., the dynamical structure factor integrated over all energies, while in experiment the data were integrated above a cutoff of $30 \mathrm{meV}$. Such a comparison is shown in Fig. 12, where we have rescaled the temperature scale, using the experimentally determined specific heat data of $\mathrm{Na}_{2} \mathrm{IrO}_{3}$ [57] which locates the high-temperature crossover in the specific heat [see Fig. 12(a)] at $T^{*}=110 \mathrm{~K}$. After this rescaling, the two data

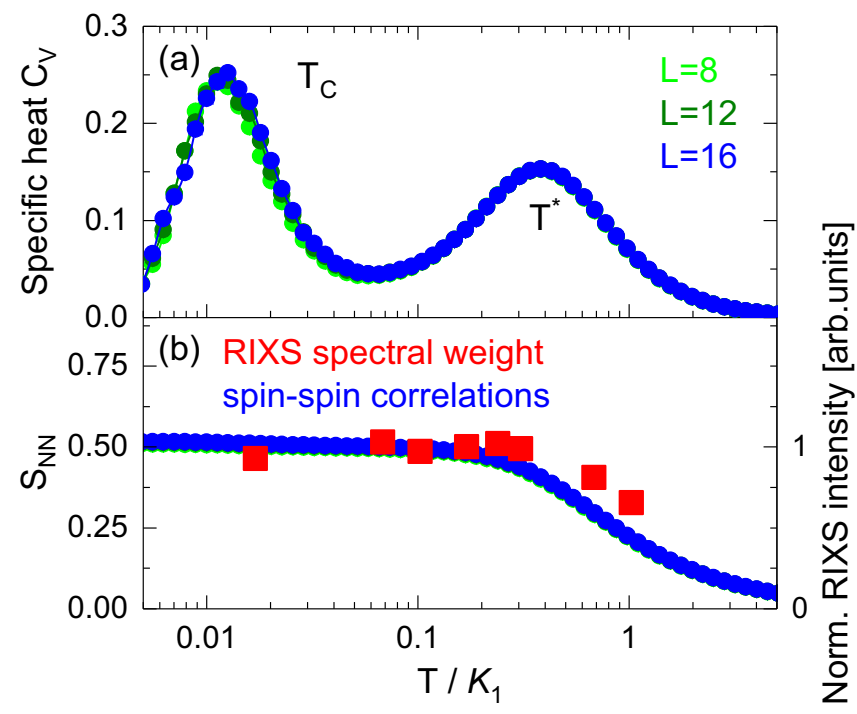

FIG. 12. Thermodynamic signatures of Kitaev physics and temperature dependence of spin-spin correlations. (a) The specific heat of the pure Kitaev model exhibits a characteristic two-peak structure, with a crossover at $T^{*} \sim K_{1}$ to a disordered Kitaev spin-liquid regime and a low-temperature transition at $T_{c} \approx K_{1} / 100$ to the Kitaev spin-liquid phase (see also Fig. 1). (b) The nearest-neighbor spinspin correlations remain nearly constant up to the high-temperature crossover scale. Numerical results for the pure Kitaev model from quantum Monte Carlo simulations (blue) are shown in comparison to the temperature dependence of the RIXS intensity of $\mathrm{Na}_{2} \mathrm{IrO}_{3}$ integrated above $30 \mathrm{meV}$ (red; cf. Fig. 4). To set the temperature scale for the comparison, we used $T^{*}=110 \mathrm{~K}$ as determined from specific heat data [57].

sets bear a striking resemblance; both traces of the nearestneighbor correlations remain nearly constant up to the order of the high-temperature crossover scale and drop only then, indicating a transition to a featureless, paramagnetic state at temperatures which are one order of magnitude higher than the experimentally observed magnetic-ordering scale.

The presence of a magnetic continuum above the longrange ordering temperature is also known for non-Kitaev systems such as quasi-one-dimensional $S=1 / 2$ Heisenberg antiferromagnets. However, such Heisenberg systems typically show a strong temperature dependence of both the correlation length and the absolute value of the dynamical structure factor [64]. This is in strong contrast with the insensitivity of the RIXS intensity observed in the honeycomb iridates, which is based on the comparable temperature insensitivity of nearest-neighbor correlations.

We note that an alternate scenario for the origin of the broad continuum in $\alpha-\mathrm{RuCl}_{3}$ has recently been put forward by Winter et al. [65], which attributes it to magnon decay within a multimagnon continuum. They showed that large anisotropic couplings in spin-orbit coupled magnets can generically lead to substantial two-magnon decay, giving rise to a broad, incoherent excitation continuum in the long-range ordered state. This raises the question of whether the magnetically ordered state or the proximate spin liquid are the better starting point for the description of the excitations in $\mathrm{Na}_{2} \mathrm{IrO}_{3}$ and $\alpha-\mathrm{Li}_{2} \mathrm{IrO}_{3}$. In our experimental RIXS data, one of the 
most striking observations is that the excitation continuum is insensitive to temperature up to at least $6 T_{N}$ and persists up to temperatures of roughly $20 T_{N}$, far above the ordered state and magnon modes. Consequently, we are led to the conclusion that we cannot explain the existence of a high-temperature quasi-2D continuum via the physics of low-temperature 3D magnons and that the scenario of a proximate spin liquid is a better starting point.

Nearest-neighbor spin-spin correlations. As discussed above for the pure Kitaev model, spin-flip RIXS creates two local visons in adjacent plaquettes as illustrated in Fig. 11. Such a probe of only the nearest-neighbor spin correlations is indeed corroborated by our experimentally observed momentum dependence; see Fig. 9. Note that this observation clearly differs from the response of other spin-liquid candidates inspected on the kagome lattice and the triangular lattice which show clear deviations from a pure $\cos ^{2}(q R / 2)$ behavior $[18,66]$ due to finite correlations between next-nearest and further neighbors. The pronounced sinusoidal shape thus provides strong evidence for the dominant role of Kitaev interactions.

The strict nearest-neighbor spin-spin correlations of the pure Kitaev model are, of course, modified by the inclusion of the necessary additional interactions. These integrabilitybreaking terms add dynamics to the previously immobile flux excitations, giving rise to correlations beyond nearest neighbors [53,67]. To quantitatively illustrate this effect, we plot results from numerical exact diagonalization calculations [68,69], similar to Ref. [67], for the (static) nearestand next-nearest-neighbor spin-spin correlations in Fig. 13. Shown are data for both the pure Kitaev model (top) and an extended Kitaev model (bottom) augmented by Heisenberg and off-diagonal spin exchanges, based on the proposal [60] by Yamaji et al. for $\mathrm{Na}_{2} \mathrm{IrO}_{3}: J_{1}=+3.2, K_{1}=-29.4$, $\Gamma_{1}^{\prime}=-3.5, J_{3}=+1.7$ in units of meV. As can be seen, these static next-nearest-neighbor correlations remain negligible at elevated temperatures, remaining at a level well below $20 \%$ of the corresponding nearest-neighbor correlations in the temperature region corresponding to the upper peak in the specific heat. For a detailed discussion of the role of the individual exchange terms on the nearest- and nextnearest-neighbor spin-spin correlations, see the Appendix. The restriction of dynamical spin-spin correlations to nearest neighbors is an outstanding property of the pure Kitaev model. Experimentally, we estimated the upper limited for further-neighbor correlations to $10 \%$; see above. We conclude that this is consistent with a realistic, extended Kitaev model.

Comparison to $\alpha-\mathrm{RuCl}_{3}$. Phenomenologically, we note that the results presented here are in full analogy with the inelastic neutron scattering data on the closely related $j=1 / 2 \mathrm{com}-$ pound $\alpha-\mathrm{RuCl}_{3}$ [17]. Both in $\alpha-\mathrm{RuCl}_{3}$ and the honeycomb iridates studied here, there is a broad excitation continuum with a maximum of intensity at the $\Gamma$ point at high energies which is robust against temperature, persisting up to roughly $20 T_{\mathrm{N}}$. Additionally, the neutron data show a nearly sinusoidal modulation along $\Gamma-K$ with a period that reflects that spinspin correlations are predominantly of nearest-neighbor type. Similar to our analysis of the RIXS data, this modulation was interpreted as a signature of the experimental proximity to the



FIG. 13. Temperature dependence of static spin-spin correlations. Comparison of the nearest- (orange) and next-nearest-neighbor (green) spin-spin correlations, $S_{N N}$ and $S_{N N N}$, as a function of temperature for (a) the pure Kitaev model and (b) an extended Kitaev model augmented by Heisenberg and off-diagonal spin exchanges as proposed [60] by Yamaji et al. for $\mathrm{Na}_{2} \mathrm{IrO}_{3}$. The next-nearest-neighbor correlations remain vanishingly small in our exact diagonalization studies for the pure Kitaev model. However, in the extended model, they are finite at zero temperature but start to decrease around the temperature scale of the low-temperature specific heat peak (blue). The dashed line indicates $300 \mathrm{~K}$, using the same scale as in Fig. 12. The shaded regions indicate the estimated error of the finite-temperature calculation.

Kitaev spin liquid. Note that in our RIXS data of $\alpha-\mathrm{Li}_{2} \mathrm{IrO}_{3}$ and $\mathrm{Na}_{2} \mathrm{IrO}_{3}$, the deviations from a pure nearest-neighbor behavior are even smaller than in $\alpha-\mathrm{RuCl}_{3}$.

\section{CONCLUSIONS}

In conclusion, we find that our experimental RIXS probes of the magnetic excitations of the honeycomb iridates $\mathrm{Na}_{2} \mathrm{IrO}_{3}$ and $\alpha-\mathrm{Li}_{2} \mathrm{IrO}_{3}$ reveal strong evidence for the emergence of a proximate Kitaev spin liquid in a broad temperature and energy regime. While the existence of dominant Kitaev exchange interactions is well accepted for the honeycomb iridates, the relevance of the Kitaev spin-liquid state and the physics associated with it has been an open issue since these compounds show long-range magnetic order at low temperature. Our results reveal the unusual properties of the magnetic excitations, marking a breakthrough in this discussion. Both at temperatures up to an order of magnitude above the magnetic ordering transition as well as for highenergy probes in the magnetically ordered ground state, we find evidence of a broad continuum of magnetic states which cannot be explained in terms of low-energy magnon modes. In addition, a thorough analysis of the (spin-flip) RIXS processes reveals that the spin-spin correlations are, within our experimental resolution, confined to nearest-neighbor sites. While extremely localized in nature, the spin-spin correlations are extremely robust against thermal fluctuations up to 
temperatures of about $300 \mathrm{~K}$. Moreover, we observe very similar behavior for $\mathrm{Na}_{2} \mathrm{IrO}_{3}$ and $\alpha-\mathrm{Li}_{2} \mathrm{IrO}_{3}$ despite their different magnetic-ordering patterns, which signals that these results on the magnetic excitations mainly reflect the common feature of dominant Kitaev exchange. While each of these experimental findings individually points toward unconventional forms of magnetism, it is in fact their combination which provides nontrivial evidence that both materials are located in close proximity to a Kitaev spin liquid. As we have comprehensively argued in this work, this proximity leaves a distinct fingerprint on the high-temperature and high-energy regimes of these materials beyond their magnetically ordered ground states.

We note that the principal findings of our RIXS experiments for the honeycomb iridates $\mathrm{Na}_{2} \mathrm{IrO}_{3}$ and $\alpha-\mathrm{Li}_{2} \mathrm{IrO}_{3}$ are in striking analogy to recent inelastic neutron scattering experiments [17] on $\alpha-\mathrm{RuCl}_{3}$. Their common phenomenology-a broad continuum of magnetic excitations and spin-spin correlations confined to nearest neighbors, which are both stable way beyond their magnetic ordering temperatures-should be taken as a concrete notion of what constitutes a "Kitaev material" beyond the existence of a dominant bond-directional exchange [14], and makes these compounds prototypical examples of this new class of materials.

Note added. Recently two complementary RIXS studies on honeycomb iridates were reported [70,71]. Vale et al. [70] studied RIXS at the $\mathrm{O} K$ edge in $\alpha-\mathrm{Li}_{2} \mathrm{IrO}_{3}$ and find a series of oxygen-based (multi)phonon modes. Compared to the $\mathrm{Ir}$ $L$ edge, the spectral weight of phonons is strongly enhanced at the $\mathrm{O} K$ edge due to the long-lived intermediate state. At the $\operatorname{Ir} L_{3}$ edge, a noteworthy phonon contribution is ruled out by our comprehensive analysis of the resonance behavior. In agreement with our discussion, the phonon contribution is much more pronounced at the $\mathrm{O} K$ edge for intermediate states that hybridize with $\operatorname{Ir} e_{g}$ states rather than $t_{2 g}$ ones. Kim et al. [71] focused on the low-energy magnon contribution in $\mathrm{Na}_{2} \mathrm{IrO}_{3}$ at the $\mathrm{Ir} L_{3}$ edge, exploring the possibilities of a new analyzer with $12 \mathrm{meV}$ resolution. Their results confirm the temperature dependence of the broad continuum and support our claims.

\section{ACKNOWLEDGMENTS}

We acknowledge funding from the Deutsche Forschungsgemeinschaft (DFG; German Research Foundation)_Projects No. 277146847, No. 247310070, and No. 107745057-CRC 1238 (Projects No. A02, B03, and C03), CRC 1143 (Project No. A05), and TRR 80 (Project No. E06), respectively, and Research Grant No. JE 748/1. The numerical simulations were performed on the JUWELS cluster at FZ Jülich and the CHEOPS cluster at RRZK Cologne.

\section{APPENDIX: CORRELATIONS IN THE EXTENDED KITAEV MODEL}

In this Appendix, we investigate the effect of spin interactions beyond the pure Kitaev model on the spin-spin correlations. We individually evaluate the effect of nearestneighbor and next-nearest-neighbor Heisenberg interactions as well as off-diagonal $\Gamma_{1}^{\prime}$ interactions, as defined in Eq. (A2), which are arguably relevant in $\mathrm{Na}_{2} \mathrm{IrO}_{3}$ [13]. We make use of a pseudofermion functional renormalization group (pfFRG) approach [72], which has previously been employed to study the Kitaev model on the honeycomb lattice augmented by nearest-neighbor [73] and further-neighbor [74] Heisenberg interactions. Recently, the method has been extended to comprise arbitrary nondiagonal two-spin interactions [75], including also interactions of $\Gamma_{1}^{\prime}$ type. The pf-FRG approach can be understood as a simultaneous large- $S$ expansion [76], typically associated with magnetically ordered phases, and large- $N$ expansion [77,78], commonly favoring spin-liquid ground states. The treatment of both channels on equal footing explains why the method can be readily employed to model the delicate interplay of magnetic order and spin-liquid physics, in both two-dimensional [72] and three-dimensional $[79,80]$ frustrated quantum spin systems.

The technique is based on a rewriting of spin operators in terms of Abrikosov fermions [81] (pseudofermions), and applying a conventional fermionic functional renormalization group (FRG) scheme [82] to the resulting strongly coupled pseudofermion system. Within the FRG scheme, it is possible to simultaneously compute the renormalization (in a Wilsonian sense) of millions of interaction parameters. The renormalization group flow of the interaction parameters is generated by the installation of a (Matsubara-)frequency cutoff $\Lambda$; the flow equations are solved numerically from the limit of infinite cutoff, which corresponds to high temperatures [79], down to zero cutoff where the fully interacting physical system at zero temperature is restored. If the system undergoes a magnetic-ordering transition at finite temperatures, the associated spontaneous symmetry breaking manifests itself in the form of a nonanalyticity in the flow of the spin-spin correlation function

$$
\chi_{i j}^{\Lambda}=\int d t\left\langle\mathbf{S}_{i}(t) \mathbf{S}_{j}(0)\right\rangle
$$

at some critical cutoff scale $\Lambda_{c}$. Properties of the system below the flow breakdown point $\Lambda_{c}$ cannot be resolved within pfFRG, but the spin-spin correlations just above the breakdown can be used as an indicator for the type of magnetic order that is about to proliferate. Note that this definition of the correlation function corresponds to the elastic $(\omega=0)$ component of the (real-space transformed) dynamic structure factor.

Our general model of interest comprises Kitaev interactions at strength $K_{1}$, nearest- and third-nearest-neighbor Heisenberg interactions of strength $J_{1}$ and $J_{3}$, respectively, as well as $\Gamma_{1}^{\prime}$ interactions. It is governed by the Hamiltonian

$$
\begin{aligned}
H= & K_{1} \sum_{\langle i, j\rangle_{1}^{\gamma}} S_{i}^{\gamma} S_{j}^{\gamma}+J_{1} \sum_{\langle i, j\rangle_{1}} \mathbf{S}_{i} \mathbf{S}_{j}+J_{3} \sum_{\langle i, j\rangle_{3}} \mathbf{S}_{i} \mathbf{S}_{j} \\
& +\Gamma_{1}^{\prime} \sum_{\langle i, j\rangle_{1}^{\gamma}} \sum_{\alpha \neq \gamma}\left(S_{i}^{\gamma} S_{j}^{\alpha}+S_{i}^{\alpha} S_{j}^{\gamma}\right),
\end{aligned}
$$

where $\langle i, j\rangle_{1}$ and $\langle i, j\rangle_{3}$ denote summations over all nearest neighbors and third-nearest neighbors, respectively, and the index $\gamma=x, y, z$ denotes the bond type. We investigate four different scenarios of spin interactions beyond the pure ferromagnetic Kitaev model $\left(K_{1}=-1\right)$. (i) Augmenting the Kitaev model with a finite $\Gamma_{1}^{\prime}=-0.25$ generates ferromagnetic spin-spin correlations on nearest, next-nearest, and further 
(a)


(b)


(c)


(d)


cutoff $\Lambda$

FIG. 14. Correlations in the extended Kitaev model. The upper row displays the real-space-resolved spin-spin correlations just above the magnetic-ordering transition. All correlations are measured with respect to the reference site indicated by the gray circle. The strength of the correlation is indicated by the size of the respective circle, while the coloring indicates the sign of the correlation; blue indicates ferromagnetic correlation and red is antiferromagnetic. The bottom row shows the average nearest-neighbor correlations $\chi_{N N}$ and next-nearestneighbor correlations $\chi_{N N N}$ (left axis), as well as their ratio (right axis). The flow breakdown (see text for details) is indicated by the shaded region. The panels are computed at different sets of coupling constants, all of which have additional ferromagnetic Kitaev coupling $K_{1}=-1$. (a) $\Gamma^{\prime}=-0.25$. (b) $J_{1}=0.5$. (c) $J_{3}=0.25$. (d) $J_{1}=0.1, J_{3}=0.05$, and $\Gamma_{1}^{\prime}=-0.25$.

neighbors. It induces a phase transition into a ferromagnetically ordered state, indicated in our pf-FRG calculations by a kink in the RG flow of the observables, below which they exhibit unphysical behavior; see Fig. 14(a). (ii) Adding sizable antiferromagnetic nearest-neighbor Heisenberg interactions $J_{1}=0.5$ drives the system into the phase of "stripy" order. Its symmetrized correlations $\chi_{i j}^{\Lambda}$, which we determine just above the phase transition at $\Lambda_{c}$, are antiferromagnetic for nearest neighbors as well as next-nearest neighbors; see Fig. 14(b). (iii) The combination of Kitaev interactions with antiferromagnetic third-nearest-neighbor Heisenberg exchange $J_{3}=$ 0.25 favors a "zigzag" ordered ground state. This configuration has ferromagnetic nearest-neighbor correlations, while the second-nearest neighbors correlate antiferromagnetically; see Fig. 14(c). (iv) Ab initio calculations [60] suggest the presence of antiferromagnetic nearest-neighbor Heisenberg interactions $J_{1} /\left|K_{1}\right| \approx 0.1$ and third-nearest-neighbor Heisenberg interactions $J_{3} /\left|K_{1}\right| \approx 0.05$ in $\mathrm{Na}_{2} \mathrm{IrO}_{3}$. We have argued that both of these couplings favor antiferromagnetic correlations on next-nearest-neighbor level. These correlations can be suppressed in the presence of finite $\Gamma_{1}^{\prime}$ interactions. At moderate values for $\Gamma_{1}^{\prime} /\left|K_{1}\right| \approx-0.25$, the next-nearestneighbor correlations thereby almost vanish; see Fig. 14(d). At smaller values of $\Gamma_{1}^{\prime}$ the suppression is less drastic, particularly at $\Gamma_{1}^{\prime} /\left|K_{1}\right| \approx 0.1$, which is the value suggested by $a b$ initio calculations; in our calculations, however, we neglect bond anisotropies in the coupling constants that are also part of the $a b$ initio theory. Nevertheless, we have qualitatively demonstrated that the presence of $\Gamma_{1}^{\prime}$ interactions in $\mathrm{Na}_{2} \mathrm{IrO}_{3}$ may play an important role in the suppression of next-nearest-neighbor correlations at temperatures above the ordering transition.
[1] C. Broholm, R. J. Cava, S. A. Kivelson, D. G. Nocera, M. R. Norman, and T. Senthil, Quantum spin liquids, Science 367, eaay0668 (2020).

[2] L. Savary and L. Balents, Quantum spin liquids: A review, Rep. Prog. Phys. 80, 016502 (2017).

[3] J. Knolle and R. Moessner, A field guide to spin liquids, Annu. Rev. Condens. Matter Phys. 10, 451 (2019).

[4] A. Kitaev, Anyons in an exactly solved model and beyond, Ann. Phys. 321, 2 (2006).

[5] K. O'Brien, M. Hermanns, and S. Trebst, Classification of gapless $\mathbb{Z}_{2}$ spin liquids in three-dimensional Kitaev models, Phys. Rev. B 93, 085101 (2016).
[6] M. Hermanns, I. Kimchi, and J. Knolle, Physics of the Kitaev model: Fractionalization, dynamical correlations, and material connections, Annu. Rev. Condens. Matter Phys. 9, 17 (2018).

[7] W. Witczak-Krempa, G. Chen, Y. B. Kim, and L. Balents, Correlated quantum phenomena in the strong spin-orbit regime, Annu. Rev. Condens. Matter Phys. 5, 57 (2014).

[8] K. Kitagawa, T. Takayama, Y. Matsumoto, A. Kato, R. Takano, Y. Kishimoto, S. Bette, R. Dinnebier, G. Jackeli, and H. Takagi, A spin-orbital-entangled quantum liquid on a honeycomb lattice, Nature (London) 554, 341 (2018).

[9] M. Abramchuk, C. Ozsoy-Keskinbora, J. W. Krizan, K. R. Metz, D. C. Bell, and F. Tafti, $\mathrm{Cu}_{2} \mathrm{IrO}_{3}$ : A New Magnetically 
Frustrated Honeycomb Iridate, J. Am. Chem. Soc. 139, 15371 (2017).

[10] However, disorder related to the $\mathrm{H}$ ions may play an essential role in $\mathrm{H}_{3} \mathrm{LiIr}_{2} \mathrm{O}_{6}$ [83-85], and the magnetism of $\mathrm{Cu}_{2} \mathrm{IrO}_{3}$ is affected by $\mathrm{Cu}^{+}-\mathrm{Cu}^{2+}$ disorder [86].

[11] S. H. Chun, J.-W. Kim, J. Kim, H. Zheng, C. C. Stoumpos, C. D. Malliakas, J. F. Mitchell, K. Mehlawat, Y. Singh, Y. Choi, T. Gog, A. Al-Zein, M. Moretti Sala, M. Krisch, J. Chaloupka, G. Jackeli, G. Khaliullin, and B. J. Kim, Direct evidence for dominant bond-directional interactions in a honeycomb lattice iridate $\mathrm{Na}_{2} \mathrm{IrO}_{3}$, Nat. Phys. 11, 462 (2015).

[12] J. G. Rau, E. K.-H. Lee, and H.-Y. Kee, Spin-orbit physics giving rise to novel phases in correlated systems: Iridates and related materials, Annu. Rev. Condens. Matter Phys. 7, 195 (2016).

[13] S. M. Winter, A. A. Tsirlin, M. Daghofer, J. van den Brink, Y. Singh, P. Gegenwart, and R. Valenti, Models and materials for generalized Kitaev magnetism, J. Phys.: Condens. Matter 29, 493002 (2017).

[14] S. Trebst, Kitaev materials, arXiv:1701.07056.

[15] B. Lake, D. A. Tennant, J.-S. Caux, T. Barthel, U. Schollwöck, S. E. Nagler, and C. D. Frost, Multispinon Continua at Zero and Finite Temperature in a Near-Ideal Heisenberg Chain, Phys. Rev. Lett. 111, 137205 (2013).

[16] A. Banerjee, C. A. Bridges, J.-Q. Yan, A. A. Aczel, L. Li, M. B. Stone, G. E. Granroth, M. D. Lumsden, Y. Yiu, J. Knolle, S. Bhattacharjee, D. L. Kovrizhin, R. Moessner, D. A. Tennant, D. G. Mandrus, and S. E. Nagler, Proximate Kitaev quantum spin liquid behavior in a honeycomb magnet, Nat. Mater. 15, 733 (2016).

[17] A. Banerjee, J. Yan, J. Knolle, C. A. Bridges, M. B. Stone, M. D. Lumsden, D. G. Mandrus, D. A. Tennant, R. Moessner, and S. E. Nagler, Neutron scattering in the proximate quantum spin liquid $\alpha-\mathrm{RuCl}_{3}$, Science 356, 1055 (2017).

[18] T.-H. Han, J. S. Helton, S. Chu, D. G. Nocera, J. A. RodriguezRivera, C. Broholm, and Y. S. Lee, Fractionalized excitations in the spin-liquid state of a kagome-lattice antiferromagnet, Nature (London) 492, 406 (2012).

[19] C. Balz, B. Lake, J. Reuther, H. Luetkens, R. Schönemann, T. Herrmannsdörfer, Y. Singh, A. T. M. Nazmul Islam, E. M. Wheeler, J. A. Rodriguez-Rivera, T. Guidi, G. G. Simeoni, C. Baines, and H. Ryll, Physical realization of a quantum spin liquid based on a complex frustration mechanism, Nat. Phys. 12, 942 (2016).

[20] Y. Kasahara, T. Ohnishi, Y. Mizukami, O. Tanaka, S. Ma, K. Sugii, N. Kurita, H. Tanaka, J. Nasu, Y. Motome, T. Shibauchi, and Y. Matsuda, Majorana quantization and half-integer thermal quantum Hall effect in a Kitaev spin liquid, Nature (London) 559, 227 (2018).

[21] T. Yokoi, S. Ma, Y. Kasahara, S. Kasahara, T. Shibauchi, N. Kurita, H. Tanaka, J. Nasu, Y. Motome, C. Hickey, S. Trebst, and Y. Matsuda, Half-integer quantized anomalous thermal Hall effect in the Kitaev material $\alpha-\mathrm{RuCl}_{3}$, arXiv:2001.01899.

[22] J. Nasu, J. Knolle, D. L. Kovrizhin, Y. Motome, and R. Moessner, Fermionic response from fractionalization in an insulating two-dimensional magnet, Nat. Phys. 12, 912 (2016).

[23] L. J. Sandilands, Y. Tian, K. W. Plumb, Y.-J. Kim, and K. S. Burch, Scattering Continuum and Possible Fractionalized Excitations in $\alpha-\mathrm{RuCl}_{3}$, Phys. Rev. Lett. 114, 147201 (2015).
[24] S. K. Choi, R. Coldea, A. N. Kolmogorov, T. Lancaster, I. I. Mazin, S. J. Blundell, P. G. Radaelli, Y. Singh, P. Gegenwart, K. R. Choi, S. W. Cheong, P. J. Baker, C. Stock, and J. Taylor, Spin Waves and Revised Crystal Structure of Honeycomb Iridate $\mathrm{Na}_{2} \mathrm{IrO}_{3}$, Phys. Rev. Lett. 108, 127204 (2012).

[25] S. Choi, S. Manni, J. Singleton, C. V. Topping, T. Lancaster, S. J. Blundell, D. T. Adroja, V. Zapf, P. Gegenwart, and R. Coldea, Spin dynamics and field-induced magnetic phase transition in the honeycomb Kitaev magnet $\alpha-\mathrm{Li}_{2} \mathrm{IrO}_{3}$, Phys. Rev. B 99, 054426 (2019).

[26] J. Kim, D. Casa, M. H. Upton, T. Gog, Y.-J. Kim, J. F. Mitchell, M. van Veenendaal, M. Daghofer, J. van den Brink, G. Khaliullin, and B. J. Kim, Magnetic Excitation Spectra of $\mathrm{Sr}_{2} \mathrm{IrO}_{4}$ Probed by Resonant Inelastic X-Ray Scattering: Establishing Links to Cuprate Superconductors, Phys. Rev. Lett. 108, 177003 (2012).

[27] H. Gretarsson, N. H. Sung, J. Porras, J. Bertinshaw, C. Dietl, J. A. N. Bruin, A. F. Bangura, Y. K. Kim, R. Dinnebier, J. Kim, A. Al-Zein, M. Moretti Sala, M. Krisch, M. Le Tacon, B. Keimer, and B. J. Kim, Persistent Paramagnons Deep in the Metallic Phase of $\mathrm{Sr}_{2-x} \mathrm{La}_{x} \mathrm{IrO}_{4}$, Phys. Rev. Lett. 117, 107001 (2016).

[28] D. Pincini, J. G. Vale, C. Donnerer, A. de la Torre, E. C. Hunter, R. Perry, M. Moretti Sala, F. Baumberger, and D. F. McMorrow, Anisotropic exchange and spin-wave damping in pure and electron-doped $\mathrm{Sr}_{2} \mathrm{IrO}_{4}$, Phys. Rev. B 96, 075162 (2017).

[29] M. Moretti Sala, V. Schnells, S. Boseggia, L. Simonelli, A. Al-Zein, J. G. Vale, L. Paolasini, E. C. Hunter, R. S. Perry, D. Prabhakaran, A. T. Boothroyd, M. Krisch, G. Monaco, H. M. Rønnow, D. F. McMorrow, and F. Mila, Evidence of quantum dimer excitations in $\mathrm{Sr}_{3} \mathrm{Ir}_{2} \mathrm{O}_{7}$, Phys. Rev. B 92, 024405 (2015).

[30] X. Lu, D. E. McNally, M. Moretti Sala, J. Terzic, M. H. Upton, D. Casa, G. Ingold, G. Cao, and T. Schmitt, Doping Evolution of Magnetic Order and Magnetic Excitations in $\left(\mathrm{Sr}_{1-x} \mathrm{La}_{x}\right)_{3} \mathrm{Ir}_{2} \mathrm{O}_{7}$, Phys. Rev. Lett. 118, 027202 (2017).

[31] M. Moretti Sala, C. Henriquet, L. Simonelli, R. Verbeni, and G. Monaco, High energy-resolution set-up for Ir $L_{3}$ edge RIXS experiments, J. Electron Spectrosc. Relat. Phenom. 188, 150 (2013).

[32] M. Moretti Sala, K. Martel, C. Henriquet, A. Al Zein, L. Simonelli, Ch. J. Sahle, H. Gonzalez, M.-C. Lagier, C. Ponchut, S. Huotari, R. Verbeni, M. Krisch, and G. Monaco, A high-energy-resolution resonant inelastic $\mathrm{x}$-ray scattering spectrometer at ID20 of the European Synchrotron Radiation Facility, J. Synchrotron Rad. 25, 580 (2018).

[33] H. Gretarsson, J. P. Clancy, Y. Singh, P. Gegenwart, J. P. Hill, J. Kim, M. H. Upton, A. H. Said, D. Casa, T. Gog, and Y.-J. Kim, Magnetic excitation spectrum of $\mathrm{Na}_{2} \mathrm{IrO}_{3}$ probed with resonant inelastic x-ray scattering, Phys. Rev. B 87, 220407(R) (2013).

[34] Y. Singh and P. Gegenwart, Antiferromagnetic Mott insulating state in single crystals of the honeycomb lattice material $\mathrm{Na}_{2} \mathrm{IrO}_{3}$, Phys. Rev. B 82, 064412 (2010).

[35] F. Freund, S. C. Williams, R. D. Johnson, R. Coldea, P. Gegenwart, and A. Jesche, Single crystal growth from separated educts and its application to lithium transition-metal oxides, Sci. Rep. 6, 35362 (2016).

[36] H. Wang and J. Zhou, Numerical conversion between the Pearson VII and pseudo-Voigt functions, J. Appl. Cryst. 38, 830 (2005). 
[37] V. Hermann, J. Ebad-Allah, F. Freund, I. M. Pietsch, A. Jesche, A. A. Tsirlin, J. Deisenhofer, M. Hanfland, P. Gegenwart, and C. A. Kuntscher, High-pressure versus isoelectronic doping effect on the honeycomb iridate $\mathrm{Na}_{2} \mathrm{IrO}_{3}$, Phys. Rev. B 96, 195137 (2017).

[38] L. J. P. Ament, M. van Veenendaal, T. P. Devereaux, J. P. Hill, and J. van den Brink, Resonant inelastic x-ray scattering studies of elementary excitations, Rev. Mod. Phys. 83, 705 (2011).

[39] X. Liu, V. M. Katukuri, L. Hozoi, W.-G. Yin, M. P. M. Dean, M. H. Upton, J. Kim, D. Casa, A. Said, T. Gog, T. F. Qi, G. Cao, A. M. Tsvelik, J. van den Brink, and J. P. Hill, Testing the Validity of the Strong Spin-Orbit-Coupling Limit for Octahedrally Coordinated Iridate Compounds in a Model System $\mathrm{Sr}_{3} \mathrm{CuIrO}_{6}$, Phys. Rev. Lett. 109, 157401 (2012).

[40] J. Kim, M. Daghofer, A. H. Said, T. Gog, J. van den Brink, G. Khaliullin, and B. J. Kim, Excitonic quasiparticles in a spinorbit Mott insulator, Nat. Commun. 5, 4453 (2014).

[41] H. Gretarsson, J. P. Clancy, X. Liu, J. P. Hill, E. Bozin, Y. Singh, S. Manni, P. Gegenwart, J. Kim, A. H. Said, D. Casa, T. Gog, M. H. Upton, H.-S. Kim, J. Yu, V. M. Katukuri, L. Hozoi, J. van den Brink, and Y.-J. Kim, Crystal-Field Splitting and Correlation Effect on the Electronic Structure of $A_{2} \mathrm{IrO}_{3}$, Phys. Rev. Lett. 110, 076402 (2013).

[42] M. Moretti Sala, K. Ohgushi, A. Al-Zein, Y. Hirata, G. Monaco, and M. Krisch, $\mathrm{CaIrO}_{3}$ : A Spin-Orbit Mott Insulator Beyond the $j_{\text {eff }}=1 / 2$ Ground State, Phys. Rev. Lett. 112, 176402 (2014).

[43] M. Rossi, M. Retegan, C. Giacobbe, R. Fumagalli, A. Efimenko, T. Kulka, K. Wohlfeld, A. I. Gubanov, and M. Moretti Sala, Possibility to realize spin-orbit-induced correlated physics in iridium fluorides, Phys. Rev. B 95, 235161 (2017).

[44] A. Revelli, M. Moretti Sala, G. Monaco, P. Becker, L. Bohatý, M. Hermanns, T. C. Koethe, T. Fröhlich, P. Warzanowski, T. Lorenz, S. V. Streltsov, P. H. M. van Loosdrecht, D. I. Khomskii, J. van den Brink, and M. Grüninger, Resonant inelastic x-ray incarnation of Young's double-slit experiment, Sci. Adv. 5, eaav4020 (2019).

[45] A. Revelli, C. C. Loo, D. Kiese, P. Becker, T. Fröhlich, T. Lorenz, M. Moretti Sala, G. Monaco, F. L. Buessen, J. Attig, M. Hermanns, S. V. Streltsov, D. I. Khomskii, J. van den Brink, M. Braden, P. H. M. van Loosdrecht, S. Trebst, A. Paramekanti, and M. Grüninger, Spin-orbit entangled $j=\frac{1}{2}$ moments in $\mathrm{Ba}_{2} \mathrm{CeIrO}_{6}$ : A frustrated fcc quantum magnet, Phys. Rev. B 100, 085139 (2019).

[46] S. Moser, S. Fatale, P. Krüger, H. Berger, P. Bugnon, A. Magrez, H. Niwa, J. Miyawaki, Y. Harada, and M. Grioni, ElectronPhonon Coupling in the Bulk of Anatase $\mathrm{TiO}_{2}$ Measured by Resonant Inelastic X-Ray Spectroscopy, Phys. Rev. Lett. 115, 096404 (2015).

[47] E. Lefrancois, A.-M. Pradipto, M. Moretti Sala, L. C. Chapon, V. Simonet, S. Picozzi, P. Lejay, S. Petit, and R. Ballou, Anisotropic interactions opposing magnetocrystalline anisotropy in $\mathrm{Sr}_{3} \mathrm{NiIrO}_{6}$, Phys. Rev. B 93, 224401 (2016).

[48] F. Ye, S. Chi, H. Cao, B. C. Chakoumakos, J. A. FernandezBaca, R. Custelcean, T. F. Qi, O. B. Korneta, and G. Cao, Direct evidence of a zigzag spin-chain structure in the honeycomb lattice: A neutron and $\mathrm{x}$-ray diffraction investigation of single-crystal $\mathrm{Na}_{2} \mathrm{IrO}_{3}$, Phys. Rev. B 85, 180403(R) (2012).
[49] M. Minola, G. Dellea, H. Gretarsson, Y. Y. Peng, Y. Lu, J. Porras, T. Loew, F. Yakhou, N. B. Brookes, Y. B. Huang, J. Pelliciari, T. Schmitt, G. Ghiringhelli, B. Keimer, L. Braicovich, and M. Le Tacon, Collective Nature of Spin Excitations in Superconducting Cuprates Probed by Resonant Inelastic X-Ray Scattering, Phys. Rev. Lett. 114, 217003 (2015).

[50] G. B. Halász, N. B. Perkins, and J. van den Brink, Resonant Inelastic X-Ray Scattering Response of the Kitaev Honeycomb Model, Phys. Rev. Lett. 117, 127203 (2016).

[51] S. M. Winter, Y. Li, H. O. Jeschke, and R. Valenti, Challenges in design of Kitaev materials: Magnetic interactions from competing energy scales, Phys. Rev. B 93, 214431 (2016).

[52] J. Knolle, D. L. Kovrizhin, J. T. Chalker, and R. Moessner, Dynamics of a Two-Dimensional Quantum Spin Liquid: Signatures of Emergent Majorana Fermions and Fluxes, Phys. Rev. Lett. 112, 207203 (2014).

[53] J. Knolle, S. Bhattacharjee, and R. Moessner, Dynamics of a quantum spin liquid beyond integrability: The KitaevHeisenberg- $\Gamma$ model in an augmented parton mean-field theory, Phys. Rev. B 97, 134432 (2018).

[54] J. Yoshitake, J. Nasu, Y. Kato, and Y. Motome, Fractional Spin Fluctuations as a Precursor of Quantum Spin Liquids: Majorana Dynamical Mean-Field Study for the Kitaev Model, Phys. Rev. Lett. 117, 157203 (2016).

[55] J. Yoshitake, J. Nasu, Y. Kato, and Y. Motome, Majorana dynamical mean-field study of spin dynamics at finite temperatures in the honeycomb Kitaev model, Phys. Rev. B 96, 024438 (2017).

[56] A. M. Samarakoon, A. Banerjee, S.-S. Zhang, Y. Kamiya, S. E. Nagler, D. A. Tennant, S.-H. Lee, and C. D. Batista, Comprehensive study of the dynamics of a classical Kitaev spin liquid, Phys. Rev. B 96, 134408 (2017).

[57] K. Mehlawat, A. Thamizhavel, and Y. Singh, Heat capacity evidence for proximity to the Kitaev quantum spin liquid in $A_{2} \mathrm{IrO}_{3}(A=\mathrm{Na}, \mathrm{Li})$, Phys. Rev. B 95, 144406 (2017).

[58] V. M. Katukuri, S. Nishimoto, V. Yushankhai, A. Stoyanova, H. Kandpal, S. Choi, R. Coldea, I. Rousochatzakis, L. Hozoi, and J. van den Brink, Kitaev interactions between $j=1 / 2$ moments in honeycomb $\mathrm{Na}_{2} \mathrm{IrO}_{3}$ are large and ferromagnetic: Insights from $a b$ initio quantum chemistry calculations, New J. Phys. 16, 013056 (2014).

[59] S. Nishimoto, V. M. Katukuri, V. Yushankhai, H. Stoll, U. K. Rößler, L. Hozoi, I. Rousochatzakis, and J. van den Brink, Strongly frustrated triangular spin lattice emerging from triplet dimer formation in honeycomb $\mathrm{Li}_{2} \mathrm{IrO}_{3}$, Nat. Commun. 7, 10273 (2016).

[60] Y. Yamaji, Y. Nomura, M. Kurita, R. Arita, and M. Imada, FirstPrinciples Study of the Honeycomb-Lattice Iridates $\mathrm{Na}_{2} \mathrm{IrO}_{3}$ in the Presence of Strong Spin-Orbit Interaction and Electron Correlations, Phys. Rev. Lett. 113, 107201 (2014).

[61] Y. Yamaji, T. Suzuki, and M. Kawamura, Numerical algorithm for exact finite temperature spectra and its application to frustrated quantum spin systems, arXiv:1802.02854.

[62] T. Suzuki and S. Suga, Effective model with strong Kitaev interactions for $\alpha-\mathrm{RuCl}_{3}$, Phys. Rev. B 97, 134424 (2018).

[63] J. Nasu, M. Udagawa, and Y. Motome, Vaporization of Kitaev Spin Liquids, Phys. Rev. Lett. 113, 197205 (2014).

[64] H. M. Rønnow, D. F. McMorrow, and A. Harrison, HighTemperature Magnetic Correlations in the 2D $S=1 / 2$ 
Antiferromagnet Copper Formate Tetradeuterate, Phys. Rev. Lett. 82, 3152 (1999).

[65] S. M. Winter, K. Riedl, P. A. Maksimov, A. L. Chernyshev, A. Honecker, and R. Valenti, Breakdown of magnons in a strongly spin-orbital coupled magnet, Nat. Commun. 8, 1152 (2017).

[66] Y. Shen, Y.-D. Li, H. Wo, Y. Li, S. Shen, B. Pan, Q. Wang, H. C. Walker, P. Steffens, M. Boehm, Y. Hao, D. L. Quintero-Castro, L. W. Harriger, M. D. Frontzek, L. Hao, S. Meng, Q. Zhang, G. Chen, and J. Zhao, Evidence for a spinon Fermi surface in a triangular-lattice quantum-spin-liquid candidate, Nature (London) 540, 559 (2016).

[67] Y. Yamaji, T. Suzuki, T. Yamada, S. Suga, N. Kawashima, and M. Imada, Clues and criteria for designing a Kitaev spin liquid revealed by thermal and spin excitations of the honeycomb iridate $\mathrm{Na}_{2} \mathrm{IrO}_{3}$, Phys. Rev. B 93, 174425 (2016).

[68] S. Sugiura and A. Shimizu, Thermal Pure Quantum States at Finite Temperature, Phys. Rev. Lett. 108, 240401 (2012).

[69] S. Sugiura and A. Shimizu, Canonical Thermal Pure Quantum State, Phys. Rev. Lett. 111, 010401 (2013).

[70] J. G. Vale, C. D. Dashwood, E. Paris, L. S. I. Veiga, M. Garcia-Fernandez, A. Nag, A. Walters, Ke-Jin Zhou, I.-M. Pietsch, A. Jesche, P. Gegenwart, R. Coldea, T. Schmitt, and D. F. McMorrow, High-resolution resonant inelastic x-ray scattering study of the electron-phonon coupling in honeycomb $\alpha-\mathrm{Li}_{2} \mathrm{IrO}_{3}$, Phys. Rev. B 100, 224303 (2019).

[71] Jungho Kim, J. Chaloupka, Y. Singh, J. W. Kim, B. J. Kim, D. Casa, A. Said, X. Huang, and T. Gog, Dynamic Spin Correlations in the Honeycomb Lattice $\mathrm{Na}_{2} \mathrm{IrO}_{3}$ Measured by Resonant Inelastic X-Ray Scattering, Phys. Rev. X 10, 021034 (2020).

[72] J. Reuther and P. Wölfle, $J_{1}-J_{2}$ frustrated two-dimensional Heisenberg model: Random phase approximation and functional renormalization group, Phys. Rev. B 81, 144410 (2010).

[73] J. Reuther, R. Thomale, and S. Trebst, Finite-temperature phase diagram of the Heisenberg-Kitaev model, Phys. Rev. B 84, 100406(R) (2011).

[74] Y. Singh, S. Manni, J. Reuther, T. Berlijn, R. Thomale, W. $\mathrm{Ku}, \mathrm{S}$. Trebst, and P. Gegenwart, Relevance of the HeisenbergKitaev Model for the Honeycomb Lattice Iridates $A_{2} \mathrm{IrO}_{3}$, Phys. Rev. Lett. 108, 127203 (2012).

[75] F. L. Buessen, V. Noculak, S. Trebst, and J. Reuther, Functional renormalization group for frustrated magnets with nondiagonal spin interactions, Phys. Rev. B 100, 125164 (2019).

[76] M. L. Baez and J. Reuther, Numerical treatment of spin systems with unrestricted spin length $S$ : A functional renormalization group study, Phys. Rev. B 96, 045144 (2017).

[77] F. L. Buessen, D. Roscher, S. Diehl, and S. Trebst, Functional renormalization group approach to $\mathrm{SU}(N)$ Heisenberg models: Real-space renormalization group at arbitrary $N$, Phys. Rev. B 97, 064415 (2018).

[78] D. Roscher, F. L. Buessen, M. M. Scherer, S. Trebst, and S. Diehl, Functional renormalization group approach to $\mathrm{SU}(N)$ Heisenberg models: Momentum-space renormalization group for the large- $N$ limit, Phys. Rev. B 97, 064416 (2018).

[79] Y. Iqbal, R. Thomale, F. Parisen Toldin, S. Rachel, and J. Reuther, Functional renormalization group for threedimensional quantum magnetism, Phys. Rev. B 94, 140408(R) (2016).

[80] F. L. Buessen and S. Trebst, Competing magnetic orders and spin liquids in two- and three-dimensional kagome systems: Pseudofermion functional renormalization group perspective, Phys. Rev. B 94, 235138 (2016).

[81] A. A. Abrikosov, Electron scattering on magnetic impurities in metals and anomalous resistivity effects, Phys. Phys. Fiz. 2, 5 (1965).

[82] C. Wetterich, Exact evolution equation for the effective potential, Phys. Lett. B 301, 90 (1993).

[83] R. Yadav, R. Ray, M. S. Eldeeb, S. Nishimoto, L. Hozoi, and J. van den Brink, Strong Effect of Hydrogen Order on Magnetic Kitaev Interactions in $\mathrm{H}_{3} \mathrm{LiIr}_{2} \mathrm{O}_{6}$, Phys. Rev. Lett. 121, 197203 (2018).

[84] Y. Li, S. M. Winter, and R. Valenti, Role of Hydrogen in the Spin-Orbital-Entangled Quantum Liquid Candidate $\mathrm{H}_{3} \mathrm{LiI}_{2} \mathrm{O}_{6}$, Phys. Rev. Lett. 121, 247202 (2018).

[85] J. Knolle, R. Moessner, and N. B. Perkins, Bond-Disordered Spin Liquid and the Honeycomb Iridate $\mathrm{H}_{3} \mathrm{LiIr}_{2} \mathrm{O}_{6}$ : Abundant Low-Energy Density of States from Random Majorana Hopping, Phys. Rev. Lett. 122, 047202 (2019).

[86] Y. S. Choi, C. H. Lee, S. Lee, S. Yoon, W.-J. Lee, J. Park, A. Ali, Y. Singh, J.-C. Orain, G. Kim, J.-S. Rhyee, W.-T. Chen, F. Chou, and K.-Y. Choi, Exotic Low-Energy Excitations Emergent in the Random Kitaev Magnet $\mathrm{Cu}_{2} \mathrm{IrO}_{3}$, Phys. Rev. Lett. 122, 167202 (2019). 